ARTICLE

\title{
Obesity exacerbates colitis-associated cancer via IL-6-regulated macrophage polarisation and CCL- 20/CCR-6-mediated lymphocyte recruitment
}

Claudia M. Wunderlich¹, P. Justus Ackermann¹, Anna Lena Ostermann', Petra Adams-Quack², Merly C. Vogt ${ }^{1}$, My-Ly Tran', Alexei Nikolajev², Ari Waisman (1) 2, Christoph Garbers ${ }^{3}$, Sebastian Theurich', Jan Mauer (1) ${ }^{4}$, Nadine Hövelmeyer ${ }^{2} \&$ F. Thomas Wunderlich ${ }^{1}$

Colorectal cancer (CRC) is one of the most lethal cancers worldwide in which the vast majority of cases exhibit little genetic risk but are associated with a sedentary lifestyle and obesity. Although the mechanisms underlying CRC and colitis-associated colorectal cancer (CAC) remain unclear, we hypothesised that obesity-induced inflammation predisposes to CAC development. Here, we show that diet-induced obesity accelerates chemically-induced CAC in mice via increased inflammation and immune cell recruitment. Obesity-induced interleukin-6 (IL-6) shifts macrophage polarisation towards tumour-promoting macrophages that produce the chemokine CC-chemokine-ligand-20 (CCL-20) in the CAC microenvironment. CCL-20 promotes CAC progression by recruiting CC-chemokine-receptor-6 (CCR-6)expressing $B$ cells and $\gamma \delta T$ cells via chemotaxis. Compromised cell recruitment as well as inhibition of $B$ and $\gamma \delta T$ cells protects against $C A C$ progression. Collectively, our data reveal a function for IL-6 in the CAC microenvironment via lymphocyte recruitment through the CCL-20/CCR-6 axis, thereby implicating a potential therapeutic intervention for human patients.

\footnotetext{
${ }^{1}$ Max Planck Institute for Metabolism Research Cologne, Institute for Genetics, University of Cologne, Cologne Excellence Cluster on Cellular Stress Responses in Aging-associated Diseases (CECAD), Center for Molecular Medicine Cologne (CMMC), Center for Endocrinology, Diabetes and Preventive Medicine (CEDP) Cologne, 50931 Cologne, Germany. ${ }^{2}$ Institute for Molecular Medicine, University Hospital Mainz, 55131 Mainz, Germany. ${ }^{3}$ Department of Biochemistry, Kiel University, Medical Faculty, 24118 Kiel, Germany. ${ }^{4}$ Department of Pharmacology, Weill Cornell Medical College, Cornell University, New York, NY 10065, USA. Correspondence and requests for materials should be addressed to F.T.W. (email: thomas.wunderlich@sf.mpg.de)
} 
T he current obesity epidemic not only accounts for the increased incidence of classical comorbidities such as type 2 diabetes mellitus, but also predisposes to the development of certain cancers-primarily those that require an inflammatory tumour microenvironment $(\mathrm{TME})^{1}$. One cancer type that is strongly associated with obesity is colorectal cancer $(\mathrm{CRC})^{2-4}$. Globally, CRC is the second most diagnosed cancer in females and the third in males with 14.1 million new cancer cases and 8.2 million deaths in $2012^{5}$.

Obesity-induced alterations in microbiota composition and stem cell modulation have been demonstrated to promote CRC development ${ }^{6,7}$, but therapeutic strategies targeting these putative drivers of CRC might have unpredictable side effects. It is wellestablished that obesity is associated with a chronic, low-grade inflammatory state ${ }^{8}$ that could also contribute to CRC development. However, the role of obesity-induced inflammation in CRC development is unknown. Importantly, obesity therapeutic strategies that reduce inflammation can be easily conducted in patients via dietary and lifestyle intervention ${ }^{9}$. Thus, reducing obesity-associated inflammation might represent a convenient strategy to prevent obesity-induced CRC.

In obesity, immune cells such as macrophages, $\mathrm{T}$ cells and $B$ cells infiltrate the white adipose tissue. Activation of these cells causes local and systemic increases of inflammatory cytokines, such as tumour necrosis factor (TNF) and interleukin (IL)-6. Elevated cytokine levels are typically associated with obesity and propagate the obesity-associated inflammatory state $^{10-13}$

IL-6 acts via its membrane-bound IL-6 receptor (IL-6R) composed of IL-6Ra that mediates specificity and the common signalling chain of IL-6-type cytokines glycoprotein 130 $(\mathrm{GP} 130)^{14}$. Though previously excluded, also ciliary neurotrophic factor (CNTF), another IL-6-type cytokine, can act as an alternative ligand for the IL-6R under certain circumstances, which might explain different outcomes when investigating IL- 6 and IL6R knockout mice ${ }^{15}$. Moreover, cell types that are not expressing IL-6R $\alpha$ can be rendered IL-6-sensitive via IL-6 transsignalling mechanisms where a soluble IL-6Ra (sIL-6Ra) is shedded from the cell surface and acts with IL- 6 on GP130-expressing cells ${ }^{16}$. Interestingly, such IL-6 transsignalling prevents obesity-induced recruitment of macrophages into adipose tissue that paradoxically failed to improve systemic insulin sensitivity ${ }^{17}$. On the other hand, enhanced central sIL-6Ra signalling improved energy and glucose homoeostasis in obesity ${ }^{18}$. Thus, different modes of signalling can affect various cell types that even do not express the necessary receptors. Moreover, we have demonstrated previously that IL- 6 exerts beneficial effects in lean mice by limiting hepatic inflammation, whereas the chronic low-grade elevation of IL-6 in obesity abrogates these functions, presumably via the development of IL-6 resistance ${ }^{19-22}$. Moreover, IL-6 signalling can polarise macrophages towards an anti-inflammatory M2 phenotype, whereas IL-6Ra deficiency leads to largely arrested macrophages in the proinflammatory M1 state ${ }^{19}$. Notably, M2 macrophages functionally overlap with tumour-associated macrophages, indicating that IL-6 might have a detrimental role in carcinogenesis ${ }^{23,24}$.

Indeed, IL-6 promotes CAC development via its action in intestinal epithelial cells (IEC) ${ }^{25-28}$. Furthermore, in the classical aetiology of CAC, the initial development of inflammatory bowel diseases (IBD) such as colitis ulcerosa and Crohn's disease are also associated with increased IL-6 level in circulation ${ }^{29}$. This suggests that induction of IL- 6 could be a common mechanism shared between obesity-induced and IBD-induced disease progression. However, how the low-grade nature of IL-6 in obesity impacts on CRC development and progression has not been investigated yet.
Here we investigate the role of obesity-induced IL-6 during development and progression of CAC in mice. We demonstrate that macrophage-specific IL-6Ra inactivation strongly ameliorates CAC in obesity. This is owing to a reduction of the chemoattractant CC-chemokine-ligand-20 (CCL-20) derived from M2 macrophages, which in turn facilitates recruitment of B cells and $\gamma \delta$ T cells into the TME in a CC-chemokine-receptor-6 (CCR-6) dependent manner. Thus, we identify IL-6R signalling in macrophages as an important mediator of colon carcinogenesis during obesity.

\section{Results}

Diet-induced obesity increases CAC development. In a first experiment, we aimed at elucidating whether diet-induced obesity affects colon inflammation and CAC. To model obesity-induced CAC in mice, we exposed cohorts of C57BL/6 mice to either normal chow (NCD) or high-fat diet (HFD) feeding from weaning on. As expected, 8-week-old HFD-fed animals exhibited increased body weight and body fat content with increased serum insulin and leptin levels as well as impaired glucose homoeostasis (Supplementary Fig. 1a-e).

Next, we investigated expression of inflammatory cytokines as well as markers for immune cells in the colon tissue of these mice. This analysis revealed the elevated expression of inflammatory cytokines Il6, Tnf, Ill $\beta$ and Il10 in the colon of obese mice compared with lean controls (Fig. 1a). Examination of immune cell markers in the obese colon revealed increases in $\mathrm{T}$ cells $(C d 3 \delta)$, regulatory $\mathrm{T}$ cells (Tregs) (forkhead-box-protein $\mathrm{P} 3$ (Foxp3)), B cells (Cd19) but similar expression of the common macrophage marker epidermal growth factor-like modulecontaining mucin-like hormone receptor-like 1 (Emr1) (Fig. 1b). Thus, HFD-feeding accelerates inflammation and lymphocytes in the colon of mice.

Next, we subjected cohorts of NCD- and HFD-fed mice to the established colitis-associated adenoma protocol induced by azoxymethane (AOM) injection directly followed by three repetitive cycles of $2.5 \%$ dextrane sodium sulphate (DSS) in the drinking water over 7 days (Supplementary Fig. 1f) ${ }^{30}$. Strikingly, obese mice either died spontaneously or had to be killed during the first DSS cycle, as these animals lost more than $20 \%$ of their initial body weight owing to symptoms associated with exaggerated colitis (Supplementary Fig. 1g, h). As the standard CAC protocol turned out to be highly lethal for obese mice, we adjusted the conditions accordingly to ensure survival of all cohorts throughout the treatment period. Therefore, we injected 8-week-old mice with AOM (day 1) and supplemented the drinking water with 1.5\% DSS from day 5 for 5 days. At day 10 of the $1.5 \% \mathrm{AOM} / \mathrm{DSS}$ protocol the animals received normal water until the end of the experiment at day 62 (non-tumour, tumour) (Fig. 1c). To examine the colitis phase animals were killed with 10 weeks of age at day 13 (colitic) and as control with 8 weeks of age at day 0 (non-colitic) of the 1.5\% AOM/DSS protocol.

Notably, lean mice that developed colitis exhibited an adapted colonic microenvironment at day 13 that resembled the inflammatory state of non-colitic HFD-fed mice (Fig. 1a, b, d, e). Strikingly, HFD in colitic mice increased Il6 expression and to a lower extent Tnf, Ill $\beta$ and $I l 10$ (Fig. 1d). Although colitic mice, independent of diet, had similar presence of $\mathrm{T}$ cells and $\mathrm{B}$ cells in colons compared with non-colitic HFD-fed colons, expression of the Treg transcription factor FoxP3 was increased suggesting a shift in T-cell composition (Fig. 1b, e). Macrophages increased only in tendency in obese colitic colons (Fig. 1e). Thus, HFD feeding mimics the inflammatory phenotype found in colitis.

Colitis compromises the gut barrier function, reflected by rectal bleeding, by mounting immune responses against commensal 

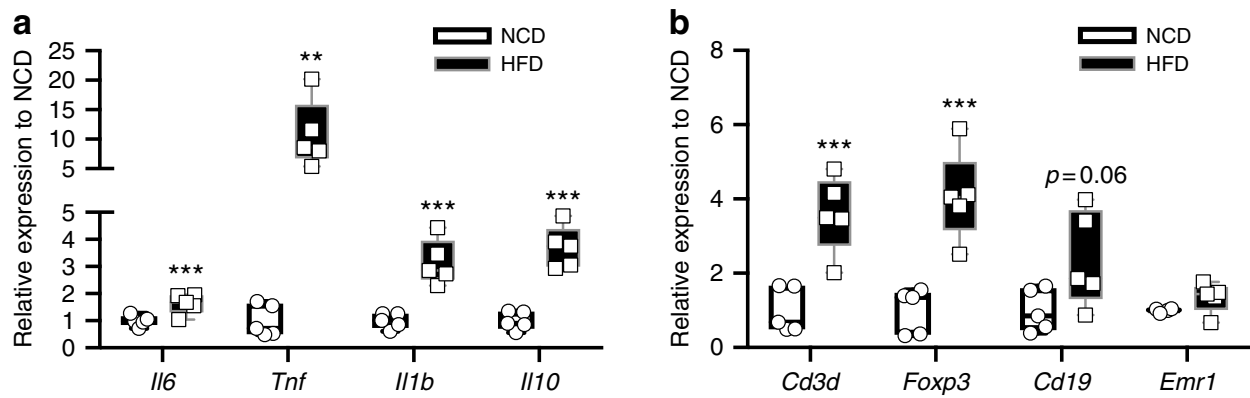

C

\section{$1.5 \%$ AOM/DSS protocol}
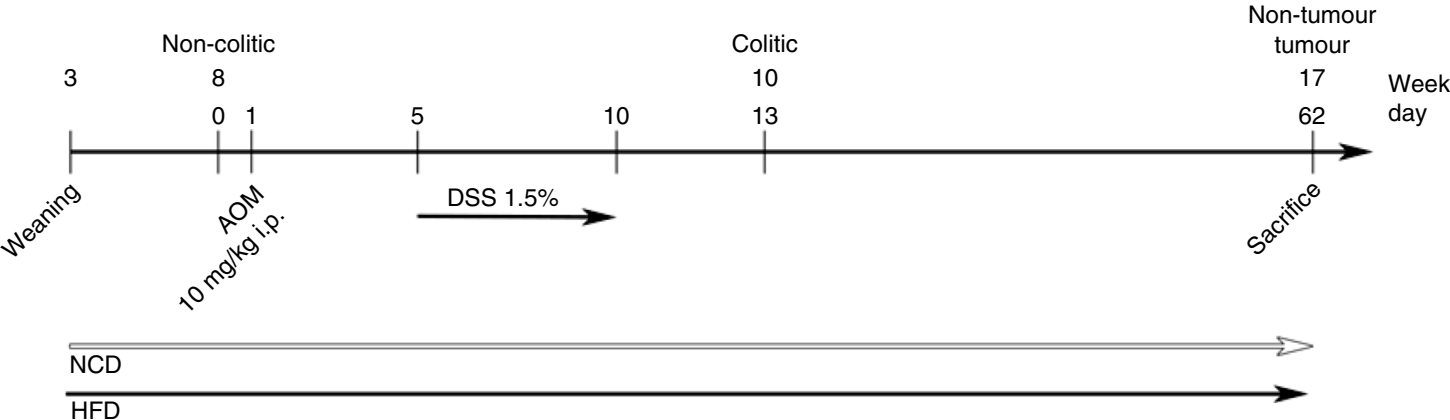

d
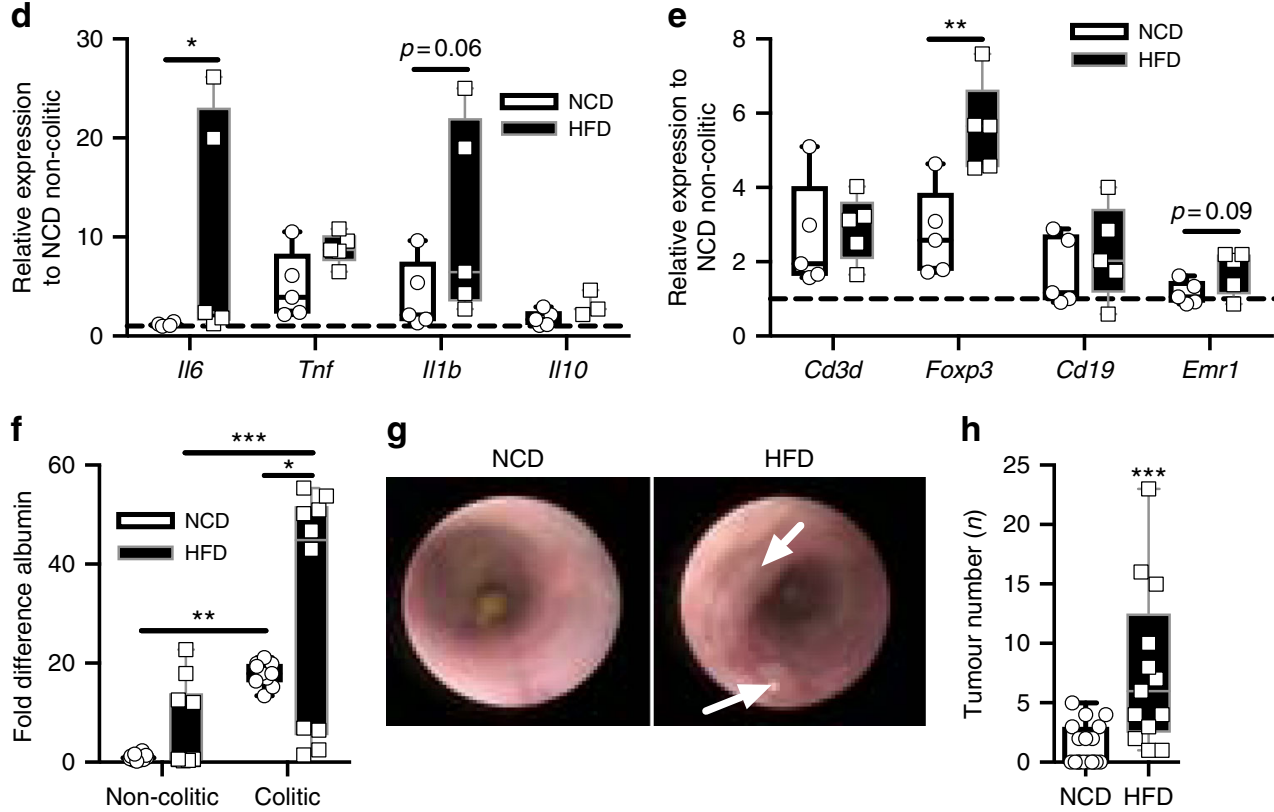

Fig. 1 Obesity enhances colonic inflammation and CAC tumour burden in C57BL/6 mice. a, b qPCR analysis of indicated gene expression in distal colons derived from 8-week-old non-colitic NCD $(n=5)$ and HFD-fed $(n=5)$ C57BL/6 mice; following results are presented relative to non-colitic NCD. c Scheme of the $1.5 \%$ AOM/DSS protocol of mice fed a NCD or a HFD from weaning (3 weeks of age) until the end of the experiment with 17 weeks of age. 8-week-old mice were injected with $10 \mathrm{mg} / \mathrm{kg}$ AOM i.p. at day 1 of the $1.5 \%$ AOM/DSS protocol. From day 5 on, mice received $1.5 \%$ DSS in the drinking water for 5 days. Mice were either killed with 8 weeks of age at day 0 (non-colitic), 10 weeks of age at day 13 for analysis of colitic conditions (colitic) or with 17 weeks of age at day 62 (non-tumour and tumour) for analysis of tumourigenesis. d, e qPCR analysis of indicated gene expression in distal colons of 10 -week-old NCD $(n=5)$ and HFD-fed $(n=5)$ colitic C57BL/6 mice; results are presented relative to non-colitic NCD. $\mathbf{f}$ Normalised albumin concentration in faeces of NCD $(n=10)$ and HFD-fed $(n=10)$ C57BL/6 mice under non-colitic (day 0$)$ and colitic (day 13) conditions. $\mathbf{g}$ Representative endoscopic pictures of 13-week-old NCD and HFD-fed C57BL/6 mice of the 1.5\% AOM/DSS protocol. h Tumour number of 17-week-old NCD ( $n=18$ ) and HFD-fed ( $n$ =13) $\mathrm{C} 57 \mathrm{BL} / 6$ mice counted at day 62 of the $1.5 \%$ AOM/DSS protocol. AOM, azoxymethane; DSS, dextran sodium sulphate, NCD, normal chow diet; HFD, high-fat diet; CAC, colitis-associated colorectal cancer. centre line: median; box limits: 1st and 3rd quartiles; whisker: maximum to minimum, ${ }^{\star} p \leq$ $0.05,{ }^{* \star} p \leq 0.01$ and ${ }^{\star \star \star} p \leq 0.001$ two-tailed unpaired Student's $t$-test $\mathbf{a}, \mathbf{b}, \mathbf{h}$ or two-way ANOVA followed by Fisher LSD $\mathbf{d}$-f 

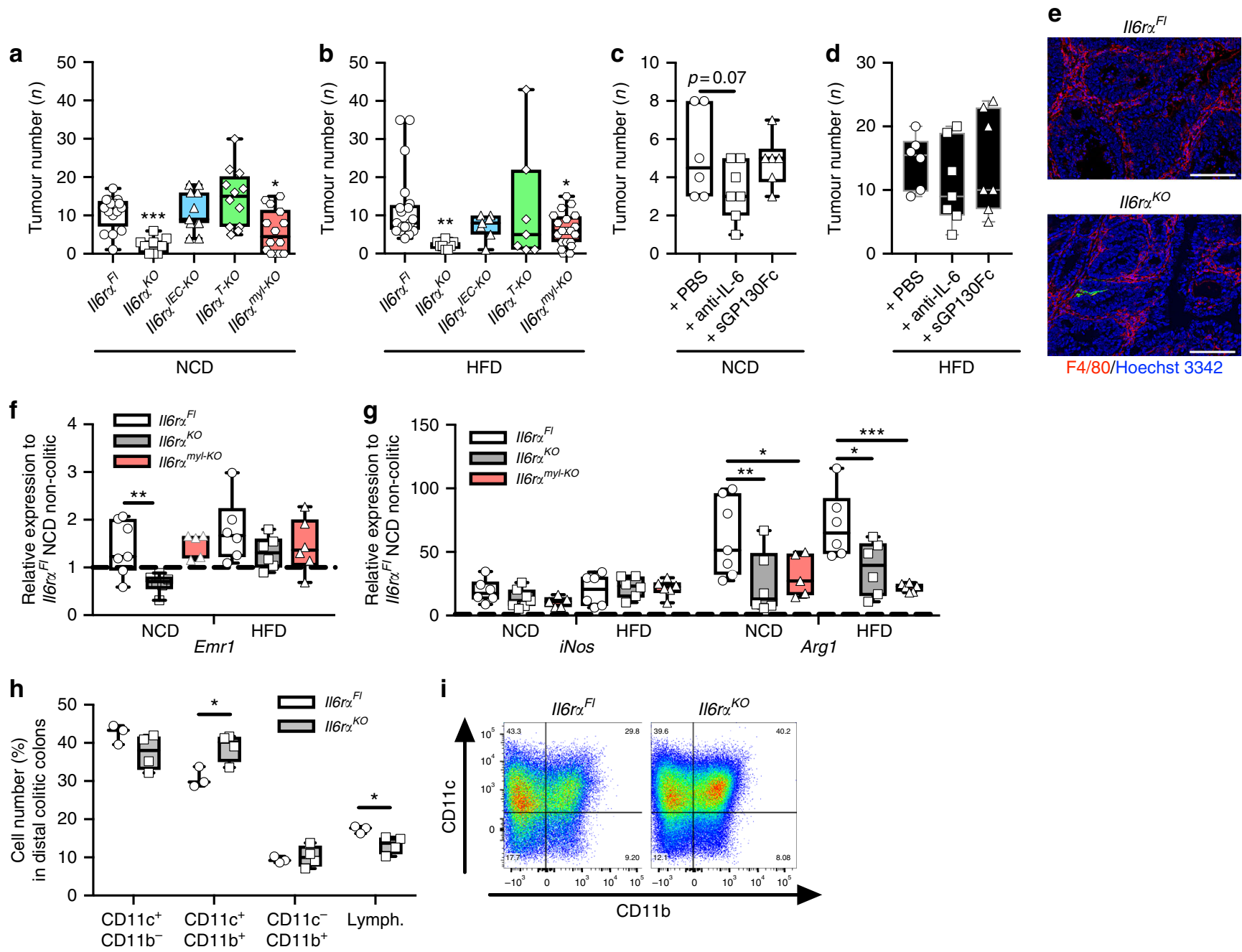

Fig. 2 IL-6R $\alpha$ deficiency in myeloid cells reduces CAC. AOM/DSS-induced tumour numbers in colons of a NCD-fed $(n=9-16)$ and $\mathbf{b}$ HFD-fed $(n=7-20)$ 17 -week-old $\left\|6 r \alpha^{F I},\right\| 6 r \alpha^{K O},\left\|6 r \alpha^{\mid E C-K O},\right\| 6 r \alpha^{T-K O}, \| 6 r \alpha^{m y l-K O}$ mice at day 62 of the $1.5 \%$ AOM/DSS protocol. Tumour numbers counted at day 62 of 17-week-old c NCD ( $n=6-7)$ or d HFD ( $n=6$-7)-fed C57BL/6 mice injected with either PBS, $500 \mu$ g anti-IL-6 antibody or $150 \mu g$ sGP130Fc at day 3 of the $1.5 \%$ AOM/DSS protocol. e Representative immunofluorescent staining of macrophages using F4/80 antibody (red) counterstained by Hoechst 3342 (blue) in colons of 17-week-old HFD-fed $116 \mathrm{r \alpha}^{\mathrm{Fl}}$ and $116 \mathrm{r \alpha} \mathrm{KO}^{\mathrm{O}}$ mice at day 62 of the $1.5 \%$ AOM/DSS protocol. $\mathbf{f}, \mathbf{g}$ qPCR analysis of indicated gene expression in tumours $(n=6-7)$ of 17 -week-old NCD or HFD-fed $\left\|6 r \alpha^{F I},\right\| 6 r \alpha^{K O}$ and $\| 6 r \alpha^{m y l-K O}$ mice at day 62 of the $1.5 \%$ AOM/DSS protocol, results are presented

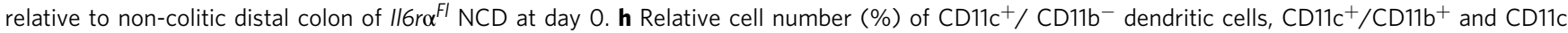
$-/$ CD $11 b^{+}$macrophages and CD11 $c^{-} /$CD11b- lymphocytes from lymphocyte gate SSC/FSC in colitic colons of NCD-fed $/ / 6 r \alpha^{F l}$ and $/ / 6 r \alpha^{K O}$ mice at day 13 of the 1.5\% AOM/DSS protocol. i Representative FACS plots of CD11c and CD11b expression in immune cells from $\mathbf{h}$. AOM, azoxymethane; DSS, dextran sodium sulphate; NCD, normal chow diet; HFD, high-fat diet; CAC, colitis-associated colorectal cancer. Centre line: median; box limits: 1st and 3rd quartiles; whisker: maximum to minimum, ${ }^{\star} p \leq 0.05,{ }^{\star \star} p \leq 0.01$ and ${ }^{\star \star \star} p \leq 0.001$ two-tailed unpaired Student's $t$-test $\mathbf{h}$, one-way a-d or two-way ANOVA followed by Fisher LSD f, g. Scale bar, $100 \mu \mathrm{m}$

factors such as bacterial lipopolysaccharides (LPS) ${ }^{30}$. To examine gut barrier function and rectal bleeding, we measured the presence of blood-abundant albumin in faeces of mice ${ }^{31}$. This analysis showed elevated immunoreactive albumin in the faeces of HFD-fed mice both under non-colitic and colitic conditions, indicating impaired gut barrier function (Fig. 1f). Further colonic investigation using endoscopy ${ }^{32}$ revealed elevated presence of dysplastic neoplasia in colitic HFD mice compared with NCD-fed mice (Fig. 1g). Importantly, macroscopic investigation of colons demonstrated an increased tumour burden in obese mice when compared with their lean littermates at day 62 (Fig. 1h and Supplementary Fig. 1i). Furthermore, colons of diet-induced obese mice were shortened, which is indicative of elevated inflammation and colitis (Supplementary Fig. 1j) ${ }^{30}$. Moreover, we observed an exaggerated immune cell infiltration into tumours of obese mice as determined by histochemistry using F4/80 and CD3 antibodies to identify macrophages and $\mathrm{T}$ cells (Supplementary Fig. 1k, 1). Taken together, diet-induced obesity exaggerated colonic inflammation, impaired gut barrier function and enhanced CAC development. Furthermore, HFD-feeding per se induced colonic inflammation, and colitis in lean mice increased inflammation to levels of non-colitic HFD-fed mice. Importantly, of the investigated cytokines, only IL-6 was upregulated in colitis of obese mice implicating that IL- 6 might have a role in obesityinduced CAC development.

IL-6R $\alpha$ deficiency in myeloid cells reduces CAC development. Next, we asked whether the obesity-induced elevation in IL-6 is driving CAC development. First, to identify the cell type that 
responds to IL-6 we induced CAC in lean and obese mice with conditional ablation of the IL-6Ra in all cells ${ }^{33}\left(I l 6 r \alpha^{K O}\right)$, in IECs $\left(I l 6 r \alpha^{I E C-K O}\right)^{27}$, in mature T cells ${ }^{34}\left(I l 6 r \alpha^{T-K O}\right)$ or in the myeloid lineage $^{19}\left(I l 6 r \alpha^{m y l-K O}\right)$ using the $1.5 \%$ AOM/DSS protocol (Fig. 1c). As a consequence of Cre recombinase expression, exons 2 and 3 of the Il6r $\alpha^{F L}$ allele are excised to conditionally inactivate
IL-6Ra in the respective cell type (Supplementary Fig. 2a-d). In line with a previous report employing IL-6 knockout mice, IL-6Ra deficiency protected against CAC development under lean and under obese conditions (Fig. 2a, b, Supplementary Fig. $2 \mathrm{e}-\mathrm{j})^{25}$. The cell type-specific inactivation of IL-6 signalling revealed that IEC-specific and $\mathrm{T}$ cell-specific inactivation of a Tumour tissue

$\left\|6 r \alpha^{F I} \quad\right\| 6 r \alpha^{K O}$ b

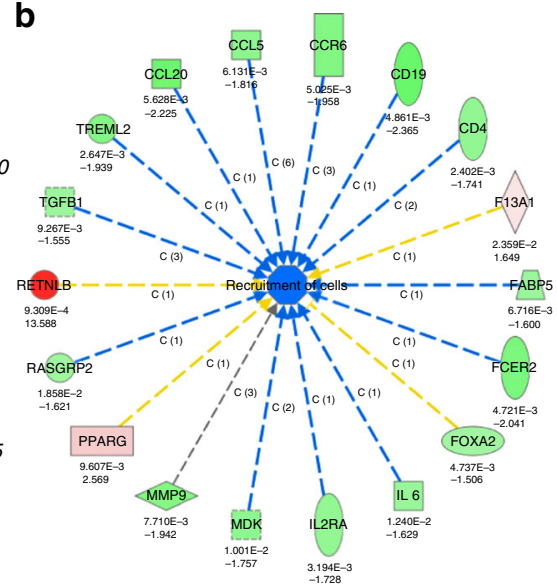

d

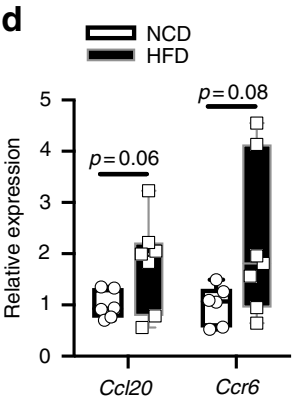

e $116 r \alpha^{F I}$

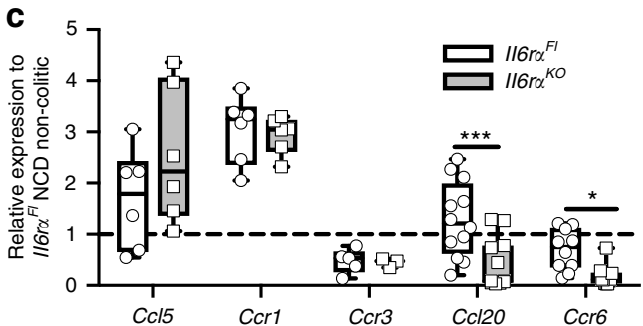

f
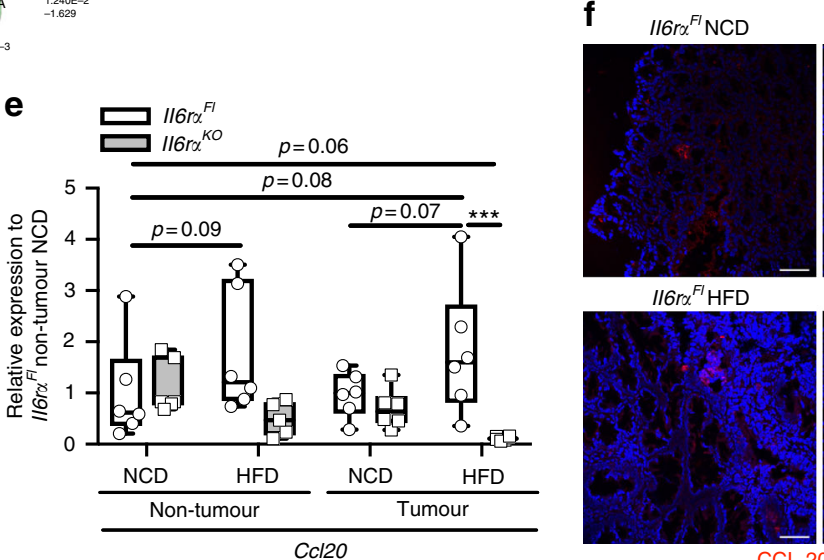

h

g

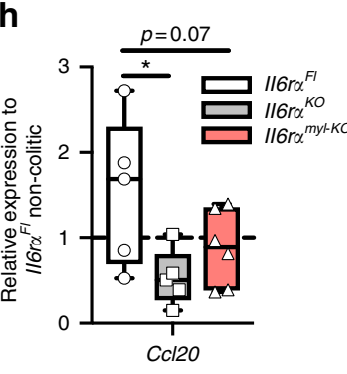

i
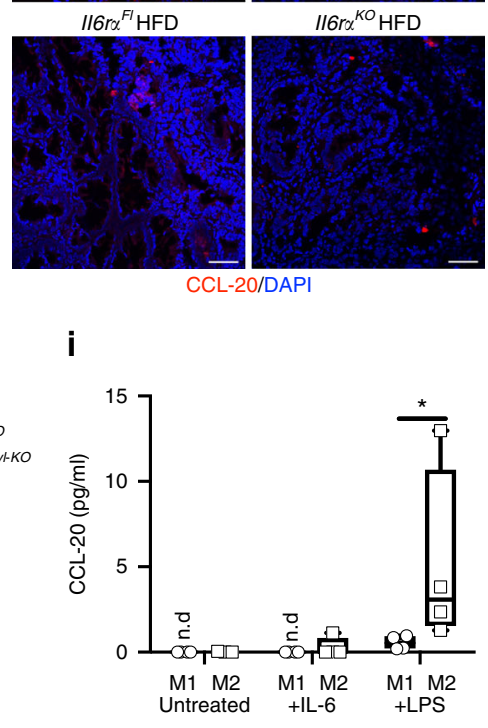

I
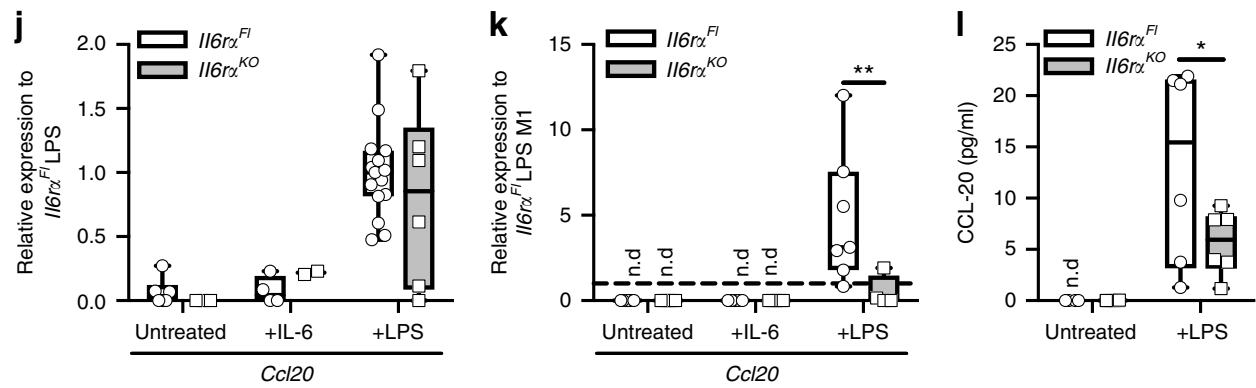
IL-6R $\alpha$ did not decrease CAC development. However, myeloid lineage-specific IL-6Ra deficiency protected against CAC (Fig. 2a, b, Supplementary Fig. 2e-j). Macrophages can provide IL-6 and sIL-6Ra in CAC, but IL-6 signalling intrinsic in macrophages might also have a role in $\mathrm{CAC}^{25}$. To investigate whether neutralising IL-6 or sIL-6Ra affects CAC, we injected anti-IL-6 antibody or the designer cytokine soluble GP130 (sGP130Fc) before the colitis phase at day 3 of the $1.5 \%$ AOM/DSS protocol in lean and obese C57BL/6 mice. C57BL/6 mice had similar body weight loss in colitis independent of anti-IL-6 and sGP130Fc treatment under NCD and HFD conditions (Supplementary Fig. $2 \mathrm{k}, \mathrm{l})$. Of note, tumour numbers were only reduced in tendency upon neutralisation with anti-IL-6 in lean and obese mice (Fig. 2c, d, Supplementary Fig. 2m-r). On the other hand, lean and obese mice with blockade of sIL-6Ra signalling had similar tumour numbers independent of neutralisation (Fig. 2c, d). However, anti-IL-6 and SGP130Fc treatment in lean mice reduced afflicted colonic area (Supplementary Fig. $2 \mathrm{~m}$ ), which is in line with a previous report, demonstrating IL- 6 deficiency reducing tumour area in $\mathrm{CAC}^{25}$. Of note, other IL-6-type cytokines such as CNTF that have not been blocked by antibody-mediated IL-6 depletion and blockade of sIL-6R $\alpha$ via sGP130Fc might compensate in CAC development. Nevertheless, neutralising IL-6 and sIL-6R $\alpha$ agents are less effective than genetic IL-6Ra ablation and in line with our previous experiments might even suggest macrophage-intrinsic IL-6 signalling functions impacting on CAC.

In this context, we have previously demonstrated that IL-6R $\alpha$ deficiency in the myeloid compartment impedes M2 macrophage polarisation $^{19}$. Furthermore, reports allocate M2-like macrophages a tumour-promoting function via angiogenic and immunomodulatory factors ${ }^{35}$. On the other hand, M1-like macrophages combat tumours via their proinflammatory and cytotoxic potential ${ }^{36}$. As we observed similar total macrophage numbers in tumour tissues independent of genotype (Fig. 2e, f), we next asked whether IL$6 \mathrm{R} \alpha$ deficiency might alter specific macrophage subsets in CAC. Thus, we examined gene expression to identify M1/inducible nitric oxide synthase (iNos)- and M2/arginase 1 (Arg1)-expressing macrophages on isolated tumour tissue derived from control, $I l 6 r \alpha^{K O}$ and Il6r $\alpha^{m y l-K O}$ mice. This analysis revealed that IL-6Ra deficiency in macrophages largely prevented M2-like polarisation as evidenced by reduced Arg1 expression, whereas iNos expression and thus M1 polarisation remained unaltered (Fig. 2g). Thus, a compromised M2 polarisation might hamper CAC in IL-6Radeficient mice. In line with this finding, fluorescence-activated cell sorting (FACS) analysis of immune cells revealed increases in $\mathrm{CD}_{11 \mathrm{c}^{+}} \mathrm{M} 1$ macrophages in colitis colons of $\mathrm{Il} 6 \mathrm{r \alpha} \mathrm{KO}^{\mathrm{KO}}$ mice, whereas the lymphocytes were reduced in Il-6ra-deficient colons (Fig. 2h, i). These findings suggest that a shifted macrophage polarisation capacity towards the inflammatory M1 lineage is contributing to the reduced CAC development found in $16 r \alpha^{K O}$ and $I l 6 r \alpha^{m y}-K O$ mice.

Altered macrophage composition might affect DSS-induced colitis, impairments of gut barrier function and proliferation of IECs by providing growth factors and cytokines. In line with this evidence, increased inflammation and IEC death in a three times DSS-mediated colitis of IL-6-deficient mice is a result of impaired IL-6-mediated signal transducer and activator of transcription 3 (Stat3) action providing survival capacities in normal and premalignant IECs ${ }^{25}$. However, colitis symptoms such as weight loss and faecal albumin were comparable between control and $I l 6 r \alpha^{K O}$ mice under NCD and HFD conditions, which might be a consequence of the mild HFD-adapted CAC protocol applied here (Supplementary Fig. 3a-d). Moreover, even colitis-associated proliferation as examined via western blot analyses against the proliferation marker proliferating cell nuclear antigen (PCNA) was similarly detectable in control and IL-6R $\alpha$-deficient samples during colitis (Supplementary Fig. 3e, f). In contrast, IL-6Radeficient tumours had less PCNA immunoreactivity compared with control tumours, whereas tumours from HFD-fed control mice had increased PCNA levels indicative of increased proliferation compared with NCD-fed controls (Supplementary Fig. 3g, h). Furthermore, decreased proliferation of IL-6Radeficient tumours was confirmed via Ki67 staining (Supplementary Fig. 3i, j). Thus, whereas colitis and colitis-associated proliferation is not affected by IL-6Ra deficiency, tumours of Il6r ${ }^{K O}$ mice exhibited less PCNA and Ki67 reactivity. Hence, the altered macrophage composition in IL-6Ra-deficient mice is not affecting the colitis phase of CAC but impacts on later stages of tumourigenesis.

IL-6R $\alpha$ deficiency attenuates M2-mediated CCL-20 expression. To gain further insights into the molecular mechanisms that promote CAC via obesity-induced IL-6, we compared global gene expression in obese control vs IL-6R $\alpha$-deficient tumour samples by microarray. Although examination of upregulated genes failed

Fig. 3 IL-6-polarised M2 macrophages express CCL-20. a Heat map of differently expressed transcripts in tumours derived from 17-week-old HFD-fed $\| 6 r \alpha^{F I}$ versus $\| 6 r \alpha^{K O}$ mice at day 62 of the $1.5 \%$ AOM/DSS protocol, assessed with a cutoff of a change in expression of 1.5-fold and $p$-value $\leq 0.025$. b IPA analysis diagram of downregulated (green) and upregulated (red) cell recruitment factors in tumour tissues derived from 17-week-old HFD-fed II6ra ${ }^{F I}$ versus $\| 6 \mathrm{ra}^{K O}$ mice at day 62 of the $1.5 \%$ AOM/DSS protocol. Upper number represent respective $p$-value, lower number fold change. c qPCR analysis of

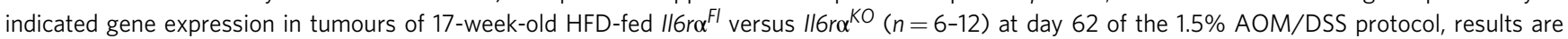
presented relative to non-colitic NCD $/ 16 \mathrm{r \alpha}^{F I}$ colons at day $0 . \mathbf{d}$ qPCR analysis of indicated gene expression in distal colons of 8-week-old NCD ( $\left.n=6\right)$ and HFD $(n=7)$ fed C57BL/6 mice. e qPCR analysis of Ccl20 expression in non-tumour tissue and tumours of 17-week-old NCD and HFD-fed $/ 16 \mathrm{ra}^{\mathrm{Fl}}$ and $/ 16 \mathrm{r \alpha} \mathrm{KO}^{\mathrm{K}}$ mice $(n=6)$ at day 62 of the $1.5 \%$ AOM/DSS protocol, results are presented relative to NCD $\| 6 r \alpha^{F l}$ non-tumour at day 62 . $\mathbf{f}$ Representative immunofluorescent stainings of CCL-20 (red) expression in colons of 17 -week-old NCD and HFD-fed $\| 6 \mathrm{ro}^{\mathrm{Fl}}$ and $\| 6 \mathrm{ra}^{\mathrm{KO}}$ mice at day 62 of the $1.5 \%$ AOM/ DSS protocol. $\mathbf{g}$ qPCR analysis of Ccr6 expression in non-tumour tissue and tumours of 17-week-old NCD and HFD-fed $\| 6 r \alpha^{F l}$ and $\| 6 r \alpha^{K O}$ mice $(n=6)$ at day 62 of the 1.5\% AOM/DSS protocol, results are presented relative to NCD $/ 16 r \alpha^{F I}$ non-tumour at day 62 . $\mathbf{h}$ qPCR analysis of CCl20 gene expression in F4/80 MACS-purified macrophages from colitic colons of 10-week-old NCD-fed $/ 16 r \alpha^{F I}, \| 6 r \alpha^{K O}$ and $\| / 6 r \alpha^{m y l-K O}(n=6)$ at day 13 of the $1.5 \%$ AOM/DSS protocol, results are presented relative to macrophages F4/80 MACS sorted from non-colitic $1 / 6$ ro ${ }^{F I}$ colons. i CCL-20 produced by M1- and M2-polarised BMDM from control mice $(n=4)$ stimulated $24 \mathrm{~h}$ with IL-6 or LPS as examined by ELISA. GPCR analysis of $C \mathrm{c} / 20$ gene expression in $\mathbf{j}$ M1 $(n=4-15)$ or $\mathbf{k}$ $\mathrm{M} 2(n=7)$-polarised BMDM stimulated with IL-6 or LPS for $8 \mathrm{~h}$ from $\| 6 r \alpha^{F I}$ and $\| 6 r \alpha^{K O}$ mice, results are presented relative to LPS-stimulated $\| 6 r \alpha^{F I}$ M1. I CCL-20 produced by M2-polarised BMDM stimulated $36 \mathrm{~h}$ with LPS from $\| 6 r \alpha^{F I}$ and $\| 6 r \alpha^{K O}$ mice $(n=6)$ as examined by ELISA. AOM, azoxymethane; DSS, dextran sodium sulphate; NCD, normal chow diet; HFD, high-fat diet; IPA, Ingenuity pathway analysis; MACS, magnetic-activated cell sorting; BMDM, bone marrow-derived macrophages; n.d., not determined. centre line: median; box limits: 1st and 3rd quartiles; whisker: maximum to minimum, ${ }^{\star} p \leq 0.05$, ${ }^{\star \star} p \leq 0.01$ and ${ }^{\star \star \star} p \leq 0.001$ two-tailed unpaired Student's $t$-test $\mathbf{d}$, one-way $\mathbf{h}$ or two-way ANOVA followed by Fisher LSD c, e, g, i, j, $\mathbf{k}, \mathbf{I}$. Scale bar, $50 \mu \mathrm{m}$ 
to clearly identify pathways, a detailed analysis of downregulated genes revealed that the TME has been altered in the absence of IL-6 signalling (Fig. 3a, Supplementary Table 1). Gene Ontology analyses revealed that cell recruitment was impaired in knockout tumours compared with controls. Here, important chemoattractants such as $\mathrm{Ccl} 20$ and $\mathrm{Ccl} 5$ were downregulated in the microarray of IL-6Ra-deficient tumours (Fig. 3b). Ccl20 and its unique receptor Ccr6 were among the 30 genes that were downregulated the highest in IL-6Ra-deficient tumours (Fig. 3a, Supplementary Table 1). However, although expression of $\mathrm{Ccl} 5$ and its receptors $C c r 1$ and $C c r 3$ were not affected by IL-6Ra deficiency in CAC, expression levels of Ccl20 and of its unique receptor Ccr6 were reduced in knockout tumours (Fig. 3c). Consistently, Ccl20 and Ccr6 were elevated in non-colitic obese
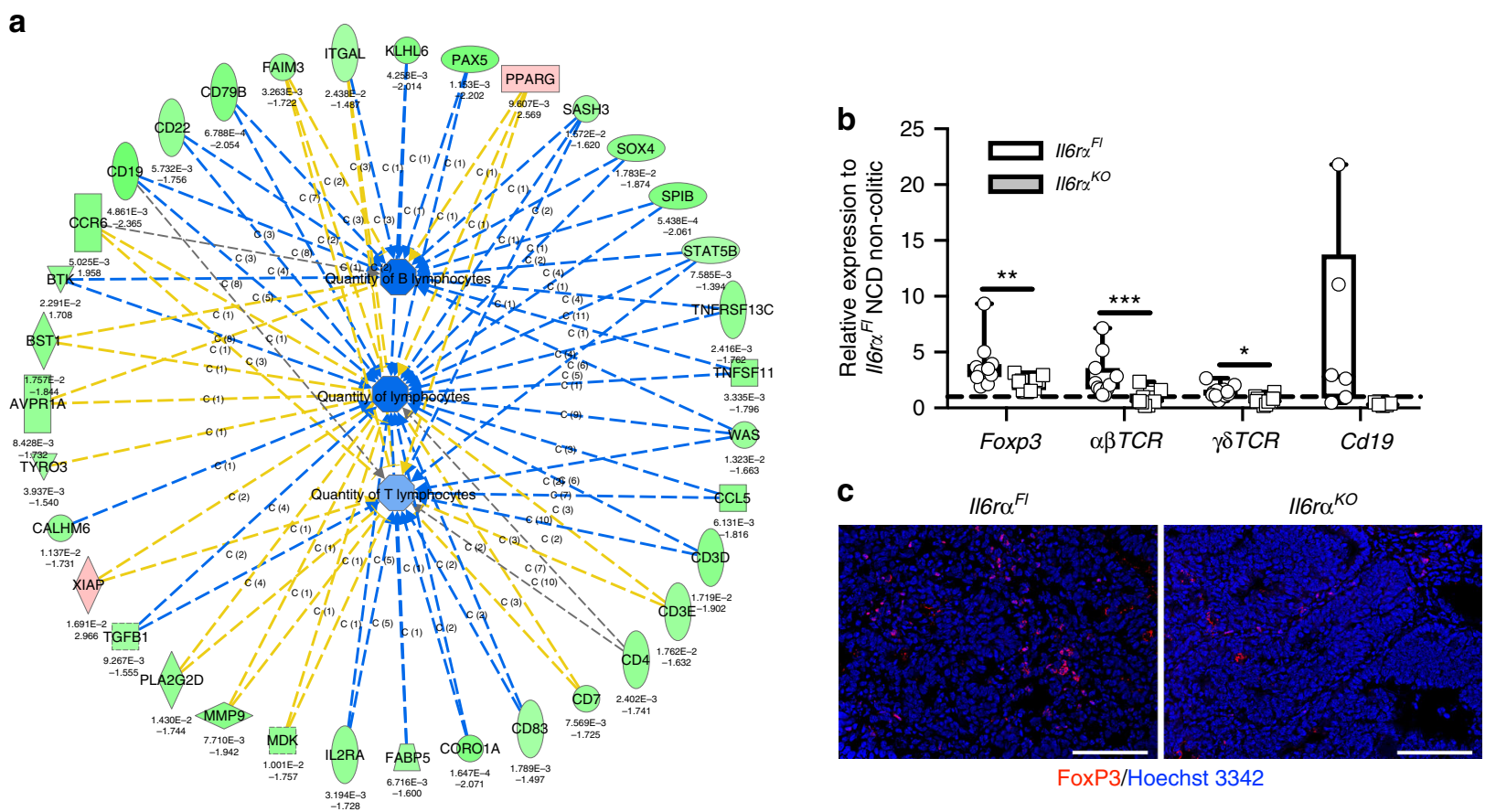

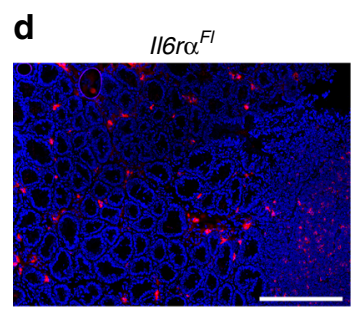

$1 / 6 r \alpha \kappa O$

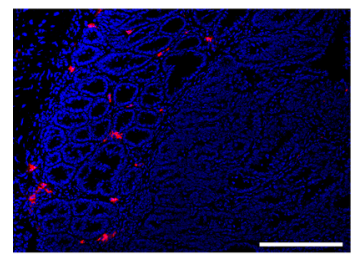

$\gamma \delta$ TCR/Hoechst 3342

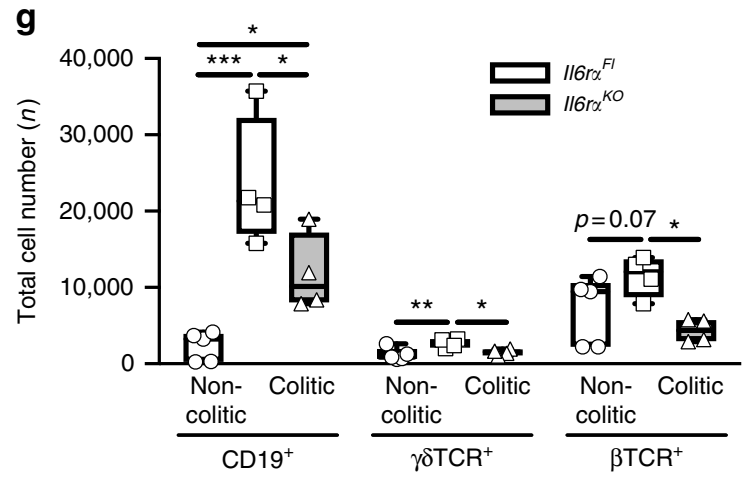

IgM/Hoechst 3342
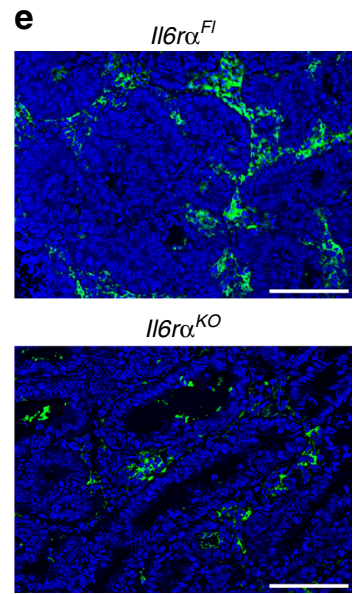

f

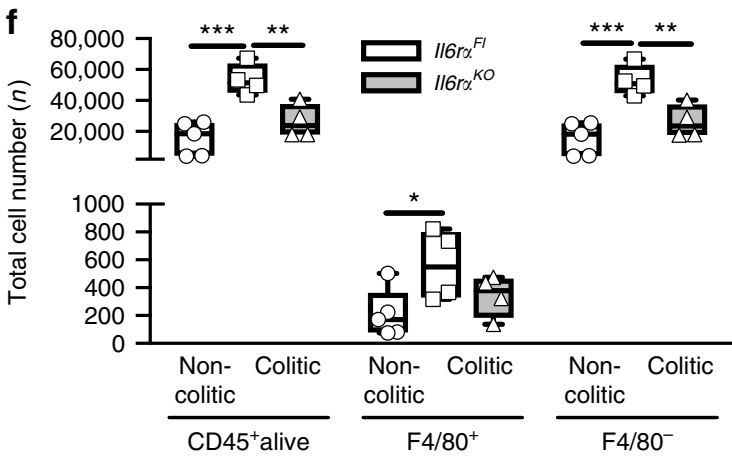

h

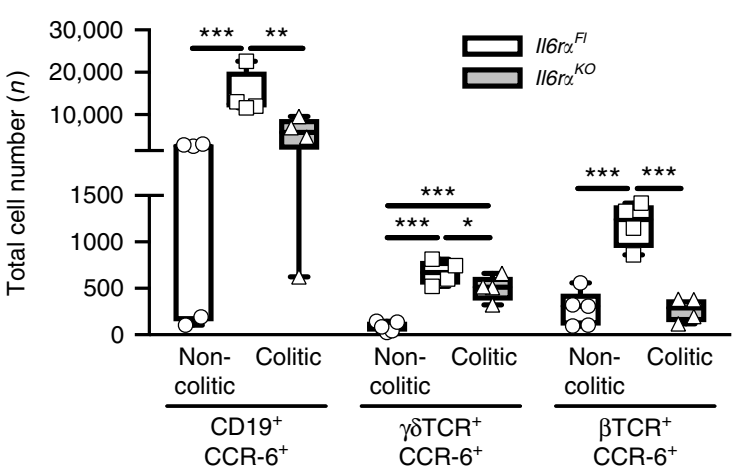


mice (Fig. 3d). Moreover, Ccl20 and Ccr6 increased in obese control tumours and were downregulated in non-tumour tissue and tumours of obese IL-6R $\alpha$-deficient mice (Fig. 3e-g). Thus, we assumed that chemoattraction of cells via the CCL-20/CCR-6 axis might affect CAC, whereas the source for CCL-20 is unclear.

CCL-20/macrophage inflammatory protein-3a derives from macrophages in numerous types of tumours, which causes the recruitment of CCR-6-expressing lymphocytes ${ }^{37-39}$. To this end, we Magnetic-activated cell sorting (MACS)-separated macrophages and IECs from colitic colons derived from control, $I l 6 r \alpha^{K O}$ and Il6r ${ }^{m y l-K O}$ mice and subjected them to qPCR to examine $\mathrm{Ccl} 20$ expression. This analysis revealed increased $\mathrm{Ccl} 20$ expression in control macrophages isolated from colitic colons when compared with IL-6Ra-deficient macrophages (Fig. 3h), whereas IL-6Ra inactivation via LysM-Cre had a tendency towards reduced $\mathrm{Ccl} 20$ expression (Fig. 3h). However, also control IEC samples exhibited increased $\mathrm{Ccl} 20$ expression in colitis either via IEC-derived Ccl20 expression or potentially via contaminating macrophages in the samples (Supplementary Fig. 4a). From our previous experiments that demonstrate IL-6 signalling in macrophages as crucial mediator in CAC, we speculated that intestinal macrophages are a main source of CCL20 in CAC. Therefore, IL-6 signalling in macrophages might either directly control CCL-20 expression on the transcriptional level or indirectly via its ability to polarise towards M2 macrophages. To test these hypotheses, we generated bone marrow-derived macrophages (BMDM) from control C57BL/6 mice and polarised them towards M1 via LPS/interferon- $\gamma$ (IFN $\gamma$ ) treatment or to M2 via IL-4/IL-6 treatment. Examination of CCL20 in the supernatant revealed that IL-6 stimulation did not directly induce CCL-20 expression in both M1 and M2 (Fig. 3i). However, LPS stimulation mimicking invading commensals increased CCL-20 in M2 compared to LPS-stimulated M1 macrophages (Fig. 3i). This experiment revealed that IL-6 indirectly controls CCL-20 expression in macrophages via its ability to polarise towards M2-type macrophages.

To confirm that IL-6Ra-deficient macrophages fail to produce CCL-20, we subjected control and Il6ra ${ }^{K O}$ BMDM to M1 and M2 protocols and stimulated them with IL-6 and LPS, respectively. Consistent with our previous finding, IL-6Ra-deficient M2 had a blunted ability to differentiate into M2 macrophages substantiated by a compromised upregulation of M2 markers Arg1 and Il4r $\alpha$ upon IL-6 stimulation (Supplementary Fig. 4b, c). Of note, M1 differentiation was similar between control and IL-6Radeficient macrophages as revealed by iNos expression (Supplementary Fig. 4d). Whereas IL-6 stimulation of M1 and M2 showed undeterminable Ccl20 expression, LPS-stimulated control M2 increased Ccl20 expression compared with the blunted response in IL-6Ra deficiency (Fig. 3j, k). Furthermore, control M2 secreted more CCL-20 compared with IL-6Radeficient BMDM in the M2 protocol under LPS-stimulated conditions (Fig. 31). Thus, CCL-20 expression in macrophages is not directly controlled via IL-6-induced transcriptional regulation but instead, IL-6 polarises macrophages in the TME towards M2 capable to produce CCL-20 upon exposure to commensal antigens such as LPS.

Collectively, these experiments suggest that CCL-20 is expressed by M2-type macrophages in colitis and that the inability of IL-6R $\alpha$-deficient macrophages to polarise towards M2-type impedes on CCL-20 expression and presumably on recruitment of CCR-6-expressing cells. Thus, the CCL-20/CCR-6 axis might recruit cell types that promote CAC development.

IL-6R $\alpha$-deficient tumours have reduced lymphocyte quantity. CCR-6, the unique receptor for CCL-20, is mainly expressed on T and $\mathrm{B}$ lymphocytes and CCR- 6 binding to CCL-20 causes chemoattraction of these cells towards the CCL-20 source ${ }^{40-43}$. In line with this fact, investigation of gene expression data from the microarray demonstrated that lymphocyte-specific genes, such as $C d 19, C d 22, C d 79 A, B$ cell activating factor receptor (Baff-R), Burton's tyrosine kinase (BTK) and paired box protein 5 (Pax5) for B cells and $C d 3 \varepsilon, C d 3 \delta, C d 4$ and Il2ra for $\mathrm{T}$ cells, were downregulated in IL-6Ra-deficient tumours (Figs. 3a and 4a, Supplementary Table 1). Furthermore, Gene Ontology analysis revealed a reduced quantity of $\mathrm{B}$ cells and $\mathrm{T}$ cells in CAC in the absence of IL-6Ra signalling (Fig. 4a). To further validate these findings, we performed qPCR and immunohistochemistry of tumours to detect Treg, $\alpha \beta \mathrm{T}$ cells, $\gamma \delta \mathrm{T}$ cells and $\mathrm{B}$ cells (Fig. 4b-e). Indeed, the qPCR experiments confirmed the reduction of these lymphocyte subsets in IL-6Ra-deficient tumours when compared with controls (Fig. 4b). Notably, these data are in line with our previous observation of reduced lymphocyte counts in IL-6R $\alpha$-deficient mice in colitis (Fig. 2h, i). To quantify CCR $-6^{+}$lymphocyte recruitment in $1.5 \%$ AOM/DSSinduced colitis, we examined total cell numbers from non-colitic and colitic control as well as IL-6R $\alpha$-deficient colons by FACS (Fig. $4 \mathrm{f}-\mathrm{h}$ ). Although consistent to our previous data, similar macrophage numbers were observed in control and IL-6Radeficient colons, IL-6Ra deficiency largely attenuated lymphocyte recruitment in colitis (Fig. 4f). Using specific antibodies in FACS to detect CD19-expressing B cells and $\gamma \delta$ as well as $\alpha \beta$ T-cell receptor-expressing $\mathrm{T}$ cells revealed that these lymphocyte subsets were reduced in the colitis colons of knockout animals (Fig. 4g). Furthermore, co-staining with CCR-6 antibody revealed that the majority of B cells and $\gamma \delta \mathrm{T}$ cells in colitis express CCR-6, whereas only a minority of $\alpha \beta$ T cells express the CCR- 6 receptor (Fig. 4h) when compared with their total numbers (Fig. 4g). Taken together, these findings clearly demonstrate the reduced

Fig. 4 IL-6R $\alpha$ deficiency compromises CCR-6-expressing lymphocyte recruitment in CAC. a IPA analysis diagram of quantity of downregulated (green) and upregulated (red) lymphocyte-specific genes in tumours derived from 17-week-old HFD-fed $/ / 6 \mathrm{ral}^{\mathrm{FI}}$ versus $/ / 6 \mathrm{r \alpha} \mathrm{KO}^{\mathrm{mice}}$ at day 62 of the $1.5 \% \mathrm{AOM} / \mathrm{DSS}$ protocol. Upper number represent respective $p$-value, lower number fold change. $\mathbf{b}$ qPCR analysis of indicated gene expression in tumours of 17 -week-old $\| 6 r \alpha^{F I}$ versus $\| 6 r \alpha^{K O}(n=6-12)$ mice at day 62 after the 1.5\% AOM/DSS protocol, results are presented relative to NCD $/ 16 r \alpha^{F I}$ non-colitic colons.

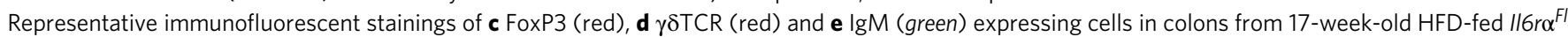
and $1 / 6 \mathrm{r} \alpha^{K O}$ mice at day 62 of the $1.5 \%$ AOM/DSS protocol counterstained with Hoechst 3342 . f Total number of CD45+, F4/80 ${ }^{+}$and F4/80- cells isolated from non-colitic and colitic colons at day 0 or day 13 of the $1.5 \%$ AOM/DSS protocol in 8 - and 10 -week-old NCD-fed $/ / 6 r \alpha^{F I}$ ( $n=4-5$ ) versus $116 r \alpha^{K O}(n=4)$ mice. $\mathbf{g}$ Total number of $\mathrm{CD} 19^{+}, \gamma \delta \mathrm{TCR}^{+}$and $\beta \mathrm{TCR}{ }^{+}$cells isolated from non-colitic and colitic colons at day 0 or day 13 of the $1.5 \%$ AOM/ DSS protocol in 8 and 10-week-old NCD-fed $/ 16 r \alpha^{F I}(n=4-5)$ versus $\| 6 r \alpha^{K O}(n=4)$ mice. h Total number of CD19+CCR-6 ${ }^{+}, \gamma \delta T C R^{+}$CCR-6 ${ }^{+}$and $\beta$ TCR ${ }^{+}{ }^{C C R}-6^{+}$cell isolated from non-colitic and colitic colons at day 0 or day 13 of the $1.5 \%$ AOM/DSS protocol in $8-$ and $10-$ week-old NCD fed $/ 16$ ro ${ }^{F /}$ ( $n=$ 4-5) versus $/ 16 r \alpha^{K O}(n=4)$ mice. AOM, azoxymethane; DSS, dextran sodium sulphate; NCD, normal chow diet; HFD, high-fat diet; IPA, Ingenuity pathway analysis; CAC, colitis-associated colorectal cancer. Centre line: median; box limits: 1st and 3rd quartiles; whisker: maximum to minimum, ${ }^{\star} p \leq 0.05$, ${ }^{\star \star} p \leq$ 0.01 and ${ }^{\star \star \star} p \leq 0.001$ two-tailed unpaired Student's $t$-test $\mathbf{b}$, one-way ANOVA followed by Fisher LSD f-h. Scale bar, $100 \mu \mathrm{m} \mathbf{c}, \mathbf{e}, 200 \mu \mathrm{m} \mathbf{d}$ 

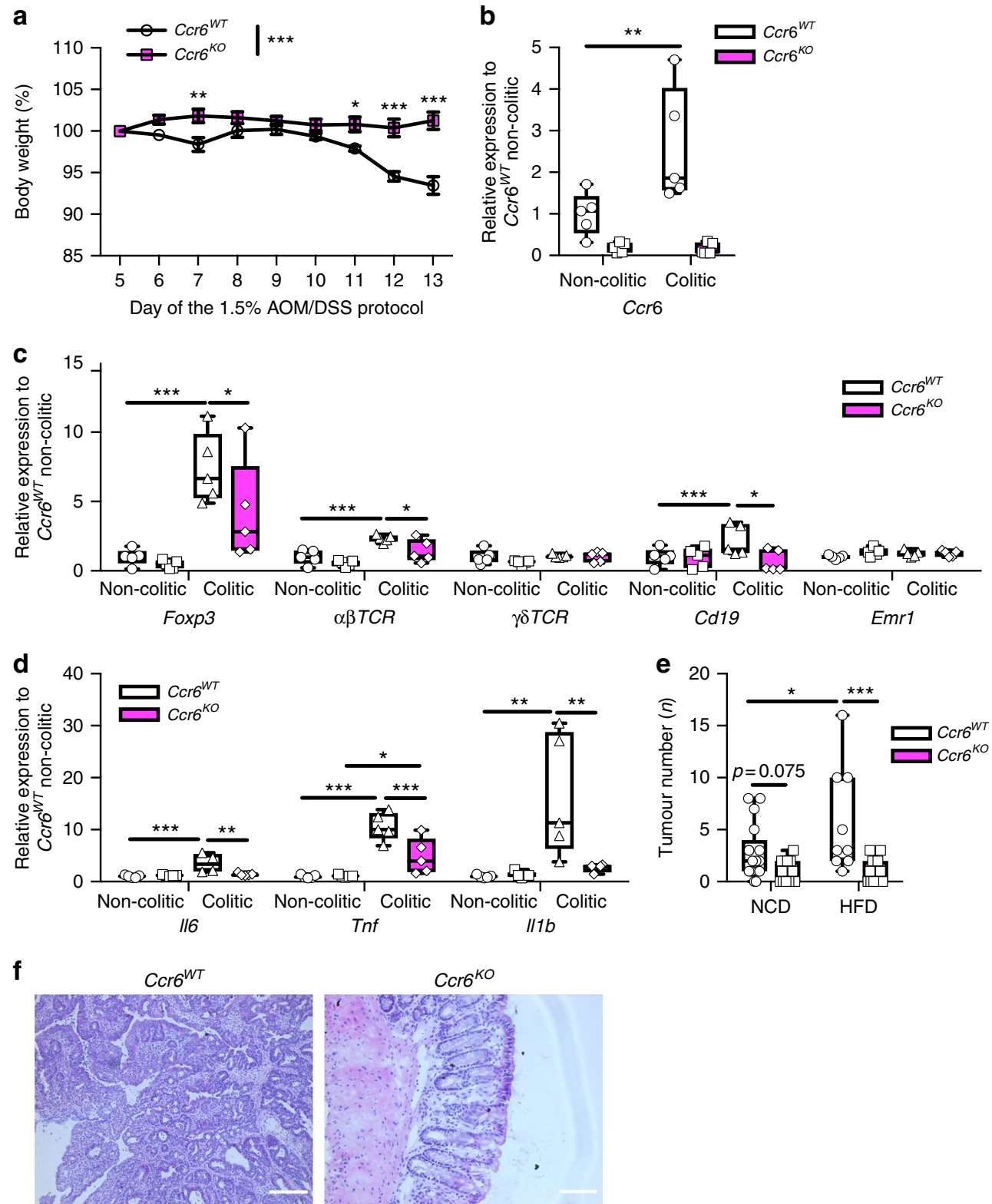

Fig. 5 CCR- $6^{+}$lymphocytes promote CAC. a Body weight loss in \% of NCD-fed Ccr6 ${ }^{W T}(n=10)$ and Ccr6 $6^{K O}$ ( $\left.n=11\right)$ mice at day 5 to day 13 of the $1.5 \%$ AOM/DSS protocol. b-d qPCR analysis of indicated gene expression in non-colitic and colitic colons of 8-and 10-week-old NCD-fed control Ccr6 ${ }^{W T}$ and $\mathrm{Ccr6}^{\mathrm{KO}}$ at day 0 and day 13 of the $1.5 \%$ AOM/DSS protocol $(n=5)$, results are presented relative to non-colitic Ccr6 ${ }^{W T}$ colons. e Tumour number of 17 week-old NCD $(n=17-13)$ and HFD ( $n=9-12)$-fed control Ccr6 $6^{W T}$ and Ccr6 ${ }^{K O}$ mice counted at day 62 of the 1.5\% AOM/DSS protocol. $\mathbf{f}$ H\&E staining of colons from NCD-fed 17-week-old Ccr6 ${ }^{W T}$ and $C c r 66^{K O}$ mice at day 62 of the 1.5\% AOM/DSS protocol. AOM, azoxymethane; DSS, dextran sodium sulphate; NCD, normal chow diet; HFD, high-fat diet; CAC, colitis-associated colorectal cancer. Data are represented as mean + SEM or centre line: median; box limits: 1st and 3rd quartiles; whisker: maximum to minimum, ${ }^{\star} p \leq 0.05,{ }^{\star \star} p \leq 0.01$ and ${ }^{\star \star \star} p \leq 0.001$ two-way ANOVA followed by Fisher LSD a-e. Scale bar, $50 \mu \mathrm{m}$

presence of CCR-6-expressing lymphocytes in IL-6Ra-deficient CAC and imply an impaired chemoattractance of these cells into the CAC TME of IL-6Ra-deficient animals.

CCR-6 ${ }^{+}$lymphocytes promote CAC development. To directly examine whether the CCL-20/CCR-6 axis is necessary for lymphocyte recruitment during colitis, we investigated CCR-6deficient mice $\left(\mathrm{Ccr} 6^{\mathrm{KO}}\right)$ in our CAC model ${ }^{44}$. During the colitis phase, CCR-6-deficient mice exhibited reduced weight loss (Fig. 5a). Importantly, colons from colitic control Ccr6 ${ }^{W T}$ mice had increased Ccr6 expression at day 13 compared with non- colitic controls, whereas consistently Ccr6 expression was absent in $C c r 6^{K O}$ mice (Fig. 5b). Colitic colons derived from $C c r 6^{K O}$ animals had reduced expression of markers for Tregs, $\alpha \beta \mathrm{T}$ cells, and B cells, but not of $\gamma \delta \mathrm{T}$ cells and macrophages (Fig. $5 \mathrm{c}$ ). Concomitantly, expression of Il6, Tnf and Ill $\beta$ were reduced in colitis colons of CCR-6-deficient mice (Fig. 5d). Thus, these analyses demonstrate that CCR-6-expressing cells are recruited during colitis and that CCR- 6 deficiency largely protects against colitis. Consistently, CCR-6-deficient animals failed to develop CAC, both under lean and obese conditions (Fig. 5e, f, Supplementary Fig. 5a-c). Therefore, we conclude that CCL-20 released in the colitis phase recruits CCR-6-expressing lymphocytes that 


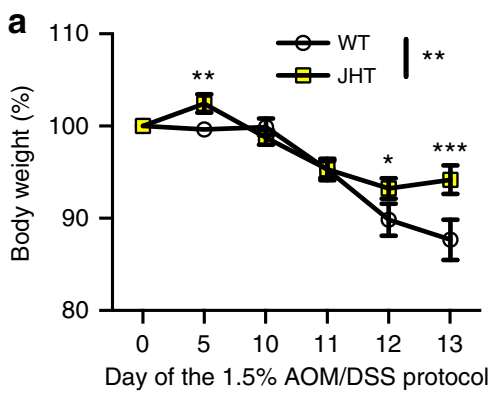

d
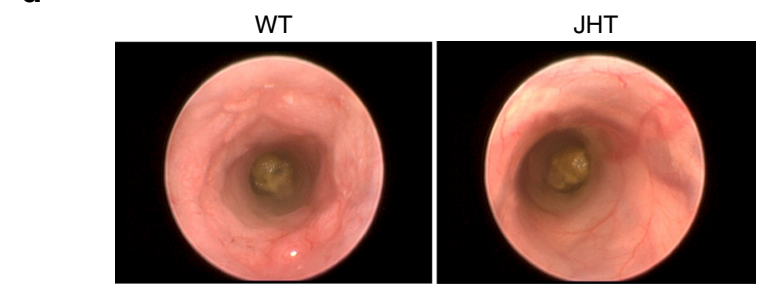

f

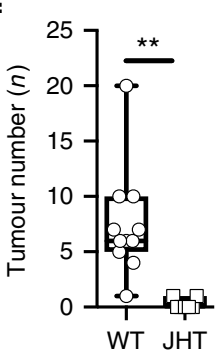

i

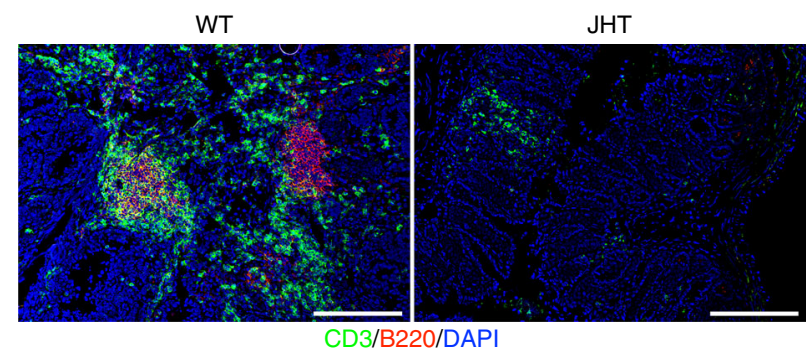

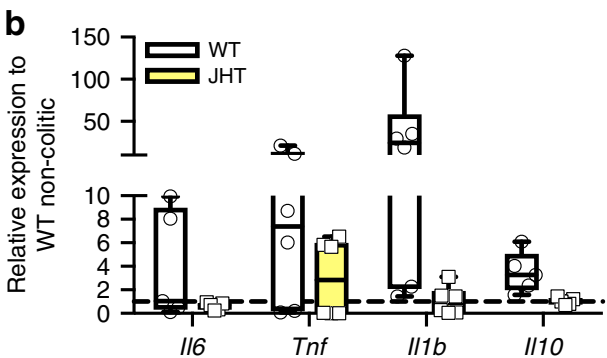

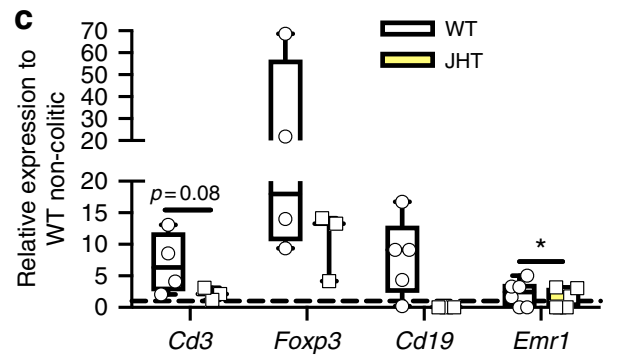

$\mathbf{e}$
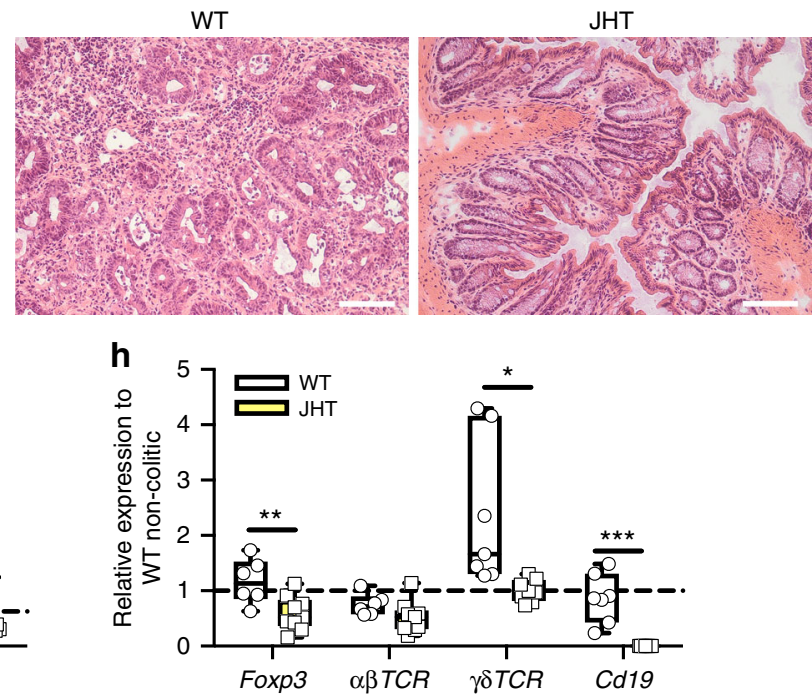

j

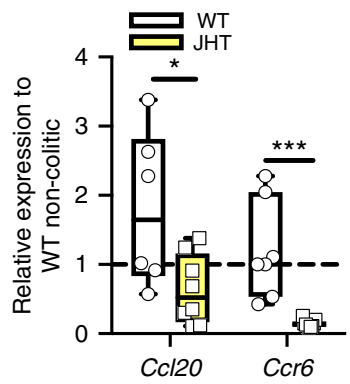

$\mathbf{k}$

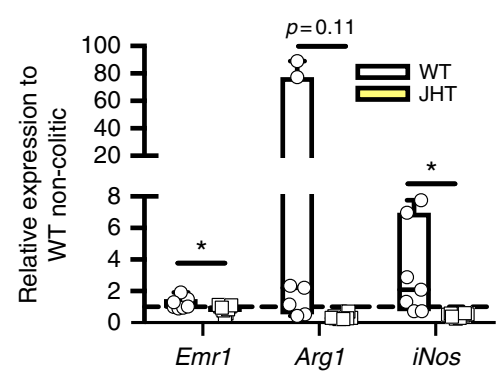

Fig. 6 Recruitment of CCR-6-expressing B cells alters the TME to promote CAC. a Body weight loss in \% of NCD-fed WT $(n=17)$ and JHT $(n=14)$ mice at day 0 to day 13 of the $1.5 \%$ AOM/DSS protocol. b, c qPCR analysis of indicated gene expression in colitic colons of 10-week-old NCD-fed WT and JHT ( $n$ =3-9) mice at day 13 of the 1.5\% AOM/DSS protocol, results are presented relative to non-colitic WT colons at day 0. $\mathbf{d}$ Representative endoscopic pictures of 13-week-old NCD-fed WT and JHT mice of the 1.5\% AOM/DSS protocol. e H\&E staining of colons from NCD-fed 17-week-old WT and JHT mice at day 62 of the 1.5\% AOM/DSS protocol. f Tumour number of 17-week-old NCD-fed WT $(n=11)$ and JHT $(n=7)$ mice counted at day 62 of the $1.5 \%$ AOM/DSS protocol. $\mathbf{g}, \mathbf{h}$ qPCR analysis of indicated gene expression in colons of 17-week-old NCD-fed WT $(n=7)$ and JHT $(n=9)$ mice at day 62 of the $1.5 \%$ AOM/DSS protocol, results are presented relative to non-colitic WT colons at day 0 . i Representative immunofluorescent stainings of CD3 (green)-expressing T cells and B220 (red)-expressing B cell in colons of 17-week-old NCD-fed WT and JHT mice at day 62 of the 1.5\% AOM/DSS protocol. j, $\mathbf{k}$ qPCR analysis of indicated gene expression in colons of 17 -week-old NCD-fed WT $(n=7)$ and JHT $(n=9)$ mice at day 62 of the $1.5 \%$ AOM/ DSS protocol, results are presented relative to non-colitic WT colons at day 0. AOM, azoxymethane; DSS, dextran sodium sulphate; NCD, normal chow diet; $\mathrm{CAC}$, colitis-associated colorectal cancer; TME, tumour microenviroment; JHT, targeted deletion of the JH locus. Data are represented as mean $+\mathrm{SEM}$ or centre line: median; box limits: 1st and 3rd quartiles; whisker: maximum to minimum, ${ }^{\star} p \leq 0.05,{ }^{\star \star} p \leq 0.01$ and ${ }^{\star \star \star} p \leq 0.001$ two-tailed unpaired Student's t-test $\mathbf{f}$ or two-way ANOVA followed by Fisher LSD $\mathbf{a}, \mathbf{b}, \mathbf{c}, \mathbf{g}, \mathbf{h}, \mathbf{j}, \mathbf{k}$. Scale bar, $50 \mu \mathrm{m} \mathbf{e}, 200 \mu \mathrm{m} \mathbf{i}$

promote CAC tumourigenesis. However, which CCR-6expressing lymphocyte population, that is recruited to the colon in colitis promotes CAC, still has to be investigated. B cells, $\gamma \delta$ $\mathrm{T}$ cells and Tregs are known to express CCR- $6^{40-43}$.

Recruitment of B cells via CCL-20/CCR-6 promotes CAC. Next, we aimed at investigating which $\mathrm{CCR}-6^{+}$cell type contributes to CAC development. A prominent CCR-6expressing lymphocyte population comprises mature B cells ${ }^{41,45}$ and $\mathrm{B}$ cells exert macrophage-polarising functions in colitis. In particular, regulatory $\mathrm{B} 1 \mathrm{~b}$-like $\mathrm{B}$ cells in the colon $\left(\mathrm{CD}^{-}, \mathrm{IgM}^{+}\right.$, CD19high, B220low) provide IL-10 to polarise macrophages towards M2-like phenotype ${ }^{46,47}$ and such B cells are induced by gut microbiota-driven IL- $1 \beta$ and IL- 6 production ${ }^{48}$. Presumably, these $\mathrm{B}$ cells sense and control commensal bacteria during 
a
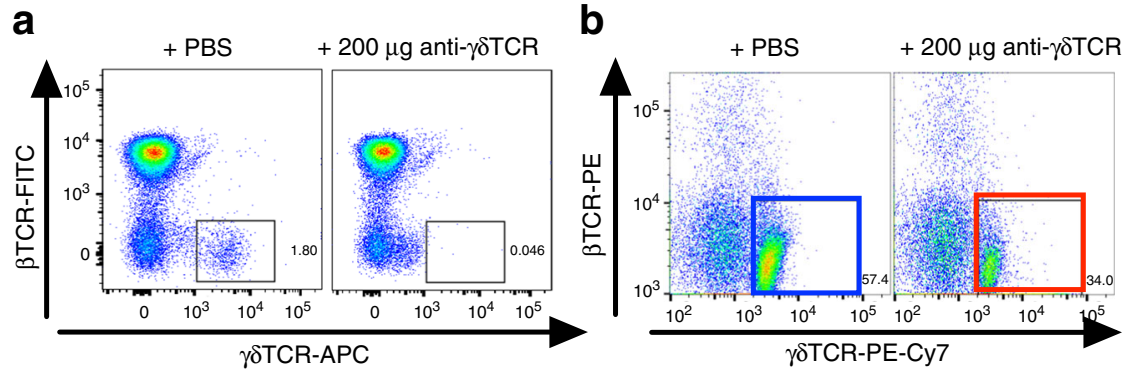

C

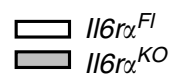

d

e
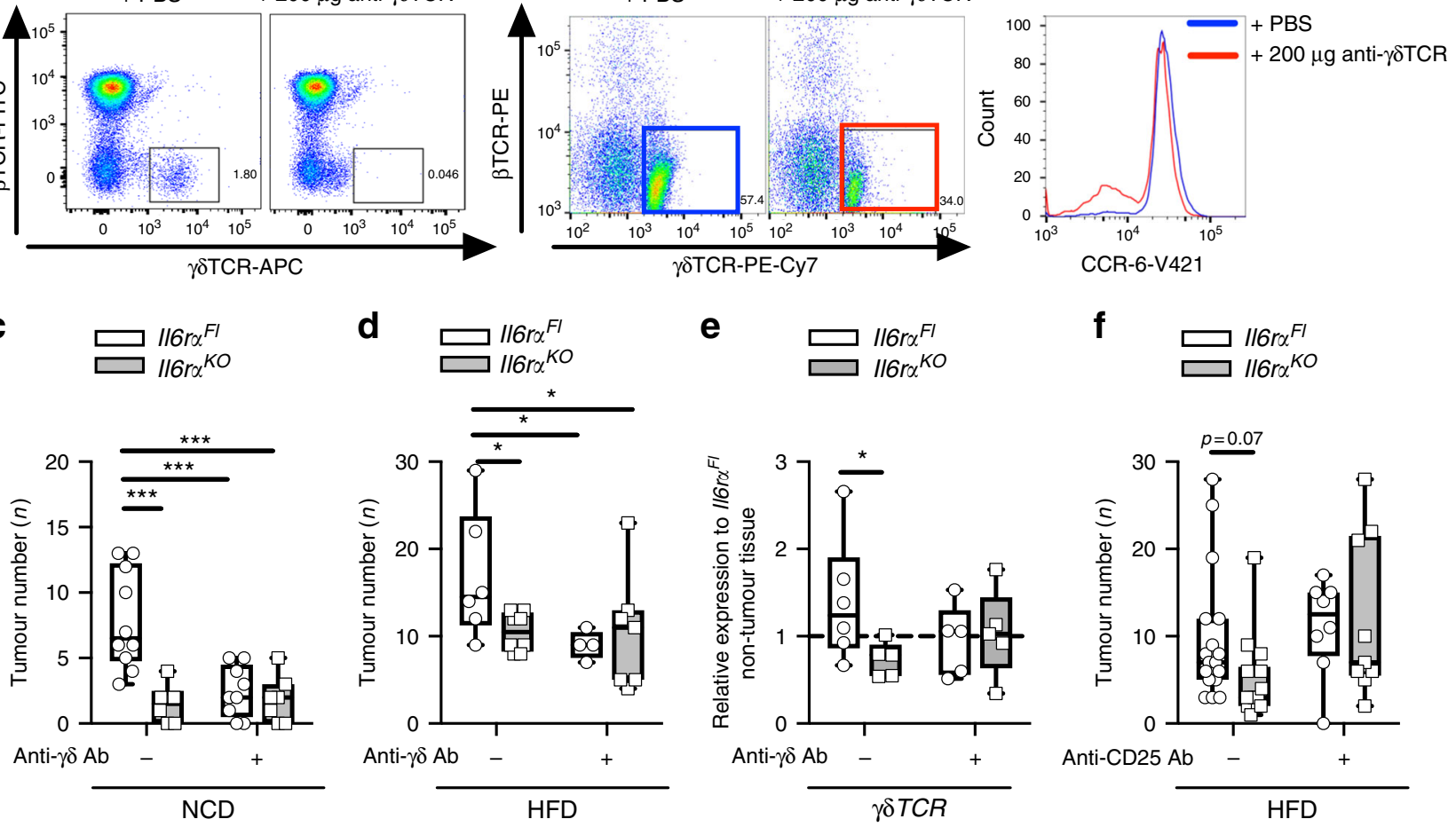

g
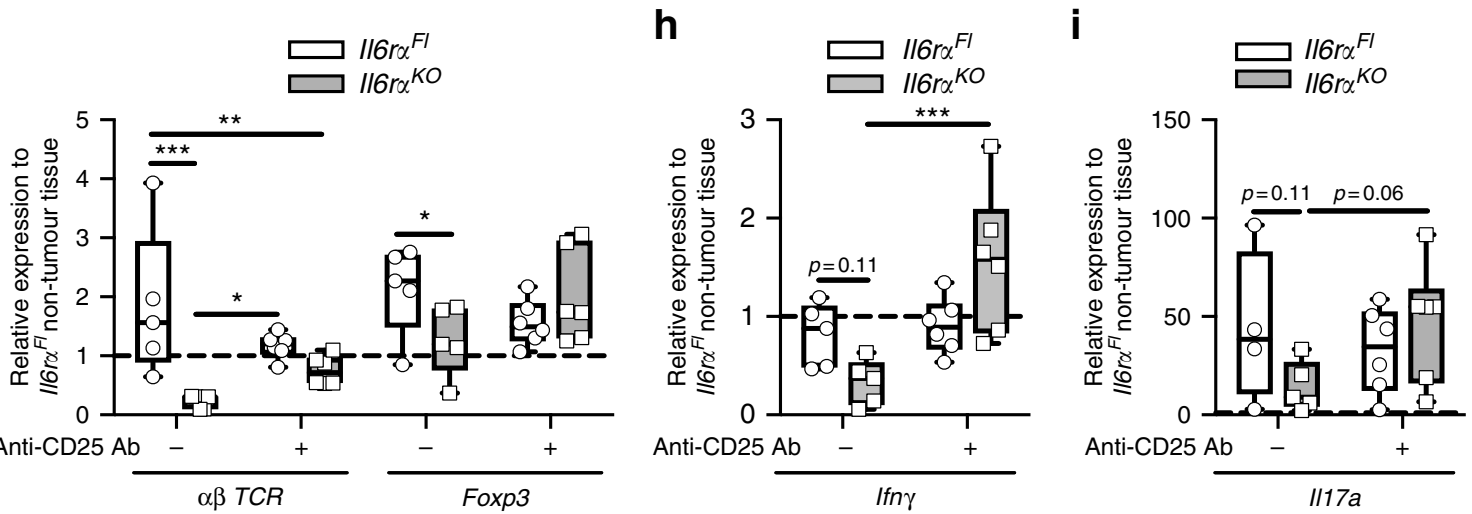

Fig. 7 Recruitment of CCR- $\sigma^{+}$T cells in CAC. a Representative FACS plots of $\beta T C R$ and $\gamma \delta T C R$ expression in blood of NCD-fed $/ 16 r \alpha^{F l}$ mice at day 5 injected either with PBS or $200 \mu \mathrm{g}$ anti- $\gamma \delta$ antibody at day 3 of the $1.5 \%$ AOM/DSS protocol. b Representative FACS plots of $\beta T C R$ and $\gamma \delta T C R$ expression in lymphocytes of colons derived from 10 -week-old NCD fed $/ 16 \mathrm{r \alpha}^{\mathrm{FI}}$ mice at day 13 injected either with PBS or $200 \mu \mathrm{g}$ anti- $\gamma \delta$ antibody at day 3 of the $1.5 \%$ AOM/DSS protocol and histogram of mean fluorescent intensity of CCR- 6 expression in $\gamma \delta T C R^{+}$lymphocytes from PBS (blue) or $200 \mu g$ anti- $\gamma \delta$ antibody (red) injected mice. Tumour number of 17-week-old NCD c $(n=6-11)$ and HFD-fed $\mathbf{d}(n=4-8)$ of $\| 6 r \alpha^{F I}$ and $\| 6 r \alpha^{K O}$ mice counted at day 62 injected with either PBS or $200 \mu \mathrm{g}$ anti- $\gamma \delta$ antibody at day 3 of the $1.5 \%$ AOM/DSS protocol. e qPCR analysis of $\gamma \delta T C R$ gene expression in tumours of $17-$ week-old NCDfed $I / 6 r \alpha^{F I}$ and $1 / 6 r \alpha^{K O}$ mice $(n=6)$ at day 62 injected with either PBS or $200 \mu \mathrm{g}$ anti- $\gamma \delta$ antibody at day 3 of the $1.5 \%$ AOM/DSS protocol, results are presented relative to $I / 6 r \alpha^{F I}$ non-tumour tissue. $\mathbf{f}$ Tumour number of 17 -week-old HFD-fed $\| 6 r \alpha^{F I}$ and $\| 6 r \alpha^{K O}$ mice $(n=8-17)$ counted at 62 days injected with either PBS or $1 \mathrm{mg}$ anti-CD25 antibody at day 3 of the 1.5\% AOM/DSS protocol. g-i aPCR analysis of indicated gene expression in tumours of $17-$ week-old HFD-fed $\| 6 \mathrm{r \alpha}^{\mathrm{FI}}$ and $\| 6 \mathrm{r \alpha} \mathrm{KO}^{\mathrm{mice}}(n=6-7)$ at day 62 injected with either PBS or $1 \mathrm{mg}$ anti-CD25 antibody at day 3 of the $1.5 \%$ AOM/DSS protocol, results are presented relative to $116 \mathrm{r}^{\mathrm{Fl}}$ non-tumour tissue. AOM, azoxymethane; DSS, dextran sodium sulphate; NCD, normal chow diet; HFD, high-fat diet; CAC, colitis-associated colorectal cancer; Ab, Antibody. centre line: median; box limits: 1st and 3rd quartiles; whisker: maximum to minimum, ${ }^{\star} p \leq 0.05,{ }^{* \star} p \leq 0.01$ and ${ }^{\star \star \star} p \leq 0.001$ two-way ANOVA followed by Fisher LSD $\mathbf{c}-\mathbf{i}$

DSS-induced colonic damage ${ }^{49}$ and engaging innate toll like receptor (TLR) 4 on B cells by LPS causes IL- 6 production ${ }^{50}$. Thus, B cells can exert numerous activities on the CAC TME that are not directly committed to humoral immunity. To investigate the role of $B$ cells and their recruitment via CCR-6/CCL-20 in CAC, we employed B cell-deficient mice. B cells rearrange their VDJ genes during $\mathrm{B}$ cell development and removal of $\mathrm{J}$ elements via gene targeting in mice results in B cell-deficient mice (JHT mice $)^{51}$. We subjected B cell-deficient JHT mice to our CAC protocol and examined colitis parameters and CAC tumour development. Body weight loss in colitis and colitis-induced expression of Il6, Tnf, Ill $\beta$ and Il10 were reduced in JHT mice (Fig. 6a, b). Furthermore, besides the lack of B cells, less immune cells such as $\mathrm{T}$ cells, Tregs and macrophages were present in colitis colons of JHT mice (Fig. 6c). This is in line with the finding that control mice developed dysplastic lesions, whereas the absence of B cells ameliorated colon pathology as revealed by endoscopy (Fig. 6d). Ultimately, control mice developed CAC, 
whereas B cell deficiency largely protected against CAC (Fig. 6e, $\mathrm{f})$. Investigation of gene expression revealed that JHT mice exhibited decreased colonic expression of Il6, Il1 $\beta$ and Il10, whereas $\operatorname{Tn} f$ was unaltered (Fig. 6g). Furthermore, not only B cells were absent in JHT colons but also gene expression markers for $\mathrm{T}$ cells and Tregs were reduced (Fig. 6h, i). Consistent with this fact was a reduced Ccr6 expression accompanied with a decreased Ccl20 expression in JHT colons, suggesting that B cells ultimately impact on the CAC TME (Fig. 6j). Apparently, the decreased Ccl20 expression is in line with an impaired macrophage polarisation to Arg1-expressing M2 as well as iNos-expressing M1-like in JHT colons (Fig. 6k). Collectively, these analyses reveal that during colitis CCL-20-recruited CCR- $6^{+} \mathrm{B}$ cells promote CAC. Contrariwise, we have also investigated JHT mice in the CAC protocol under obese conditions where $\mathrm{B}$ cell-deficient mice died either spontaneously or had to be killed owing to excessive colitis symptoms (Supplementary Fig. 5d, e). This discrepancy between diets in B cell-deficient mice hints to a more complex and dual role for B cells in colonic inflammation under different dietary exposures than hitherto assumed and even more strengthens our finding that CCR-6-expressing B cells affect CAC.

$\gamma \delta$ T-cell inhibition ameliorates CAC. Although we have demonstrated a crucial function of CCR-6-expressing B cells in the CAC TME, also T cells such as $\gamma \delta$ T cells express the CCR-6 receptor. $\gamma \delta$ T cells can be separated into CCR-6- and NK1.1expressing subtypes that fulfil $\mathrm{T}$ helper (Th) 17 and Th1 cell functions, respectively ${ }^{43}$. Koenecke et al. demonstrated that anti$\gamma \delta$ T-cell receptor (TCR) antibody injection in mice yielded inhibition of $\gamma \delta$ T cells because they downregulate their $\gamma \delta \mathrm{TCR}^{52}$. We aimed at addressing the function of $\gamma \delta \mathrm{T}$ cells and their chemoattraction via CCL-20 in our CAC model by injecting anti$\gamma \delta \mathrm{TCR}$ at day 3 of the $1.5 \% \mathrm{AOM} / \mathrm{DSS}$ protocol prior to the colitis phase of CAC into control and IL-6Ra-deficient animals. Anti- $\gamma \delta$ TCR injection led to downregulation of $\gamma \delta$ TCR as obvious from the appearance of $\mathrm{CD}^{+} \alpha \beta$ T cells and $\gamma \delta \mathrm{T}$ cells in the blood of these mice (Fig. 7a). Although most $\gamma \delta \mathrm{T}$ cells express CCR-6 during colitis in untreated mice, a proportion of $\gamma \delta$ T cells in antibody-treated mice were negative for CCR-6 expression (Fig. 7b). Ultimately, lean control mice developed CAC, whereas $\gamma \delta$ TCR antibody injection drastically reduced tumour burden in control mice similar to IL-6Ra-deficient mice (Fig. 7c, Supplementary Fig. 6a-c). Notably, the reduced CAC burden in anti$\gamma \delta$ TCR-treated control mice was largely recapitulated under obese conditions (Fig. 7d, Supplementary Fig. 6d-f). Analysis of tumour-derived gene expression revealed slightly reduced $\gamma \delta T C R$ upon anti- $\gamma \delta$ TCR antibody injection in lean mice (Fig. 7e). Our experiments are in line with previous reports assigning $\gamma \delta$ T cells as crucial mediators in colonic inflammation by providing pathogenic IL- $17^{53}$. Thus, recruitment of CCR- $6^{+} \gamma \delta \mathrm{T}$ cells promotes CAC and inhibition of $\gamma \delta$ T cells might be a reasonable therapeutically strategy against CAC.

Treg depletion restores CAC in obese IL-6Ra-deficient mice. Another CCR-6-expressing $\mathrm{T}$ lymphocyte population that has been demonstrated to contribute to CAC are Treg cells, which control gut inflammation via their immunosuppressive functions ${ }^{37}$. We have shown that IL-6 signalling in effector T cells is required to release them from Treg-mediated suppression using the well-established Ovalbumin (OVA) model ${ }^{34}$. Suppression of effector function in IL-6Ra-deficient cells included a blunted Th1/ IFN $\gamma$ and total absence of Th17 IL-17a response. However, the Th1 response could be restored in IL-6R $\alpha$-deficient T cells when Tregs were depleted by anti-CD25 antibody treatment. In order to examine whether Tregs also suppress effector functions in lean and obese IL-6Ra-deficient mice in our CAC model, anti-CD25 antibody was i.p. injected at day 3 of the $1.5 \%$ AOM/DSS protocol just prior to the colitis phase of CAC. This experimental procedure should provide only a transient Treg depletion during the colitis phase and should affect $\mathrm{T}$ effector cells to a lesser extent that upon activation upregulate CD25. Most likely, the single anti-CD25 antibody injection depletes Tregs only until anti-CD25 antibody is consumed/degraded where in the context of proceeding T-cell development these cells are able to repopulate the colon. Nevertheless, although in lean mice, anti-CD25 antibodymediated transient depletion of Treg cells exhibited only minor effects, it restored CAC development in obese IL-6Ra-deficient animals, whereas obese control mice had unaltered CAC (Fig. 7f, Supplementary Fig. $6 \mathrm{~g}-\mathrm{m})$. Detection of markers for $\alpha \beta \mathrm{T}$ cells and Treg cells in tumours of the obese cohorts revealed a reduction of these cells in IL-6R $\alpha$-deficient mice that was largely unaltered when mice received anti-CD25 treatment (Fig. 7g). However, despite the fact that also other cells express If $n \gamma$ and Il17a, the examination of T-cell effector molecules revealed decreased If $n \gamma$ and Il17a expression in obese IL-6Radeficient tumours that have been restored upon Treg depletion (Fig. 7h, i). Thus, Treg depletion in obese IL-6Ra-deficient mice restores $\mathrm{CAC}$ development via restoration of $\mathrm{T}$-cell effector functions.

Collectively, these experiments reveal a crucial role for the recruitment of CCR-6-expressing lymphocytes into the colon during the colitis phase to promote CAC. Knockout and antibody-inhibition/depletion models demonstrate that recruitment of CCR-6-expressing B cells and $\gamma \delta$ T cells promote CAC development whereas CCR- $6^{+}$Tregs exert an immunosuppressive role in CAC under obese conditions by interfering with T-cell effector functions. B cells and IL-6 synergise in the polarisation towards CCL-20-expressing macrophages causing a vicious circle of CCR-6 ${ }^{+}$lymphocyte recruitment in CAC (Fig. 8). Overall, our

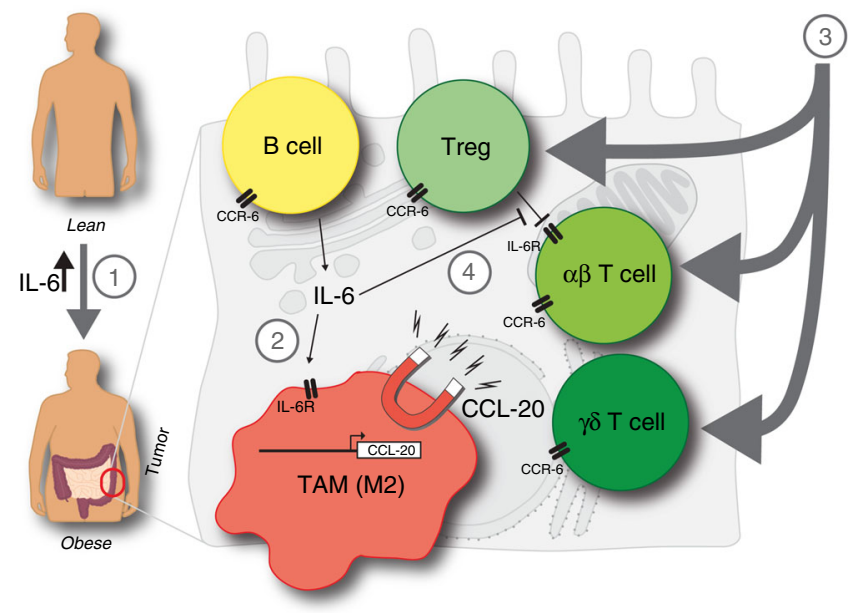

Fig. 8 Mechanism how obesity and IL-6 alter the colitis-associated tumour microenvironment. 1. Obesity-induced gut barrier defects cause a lowgrade inflammation including increases in IL-6 levels. 2. IL-6 polarises macrophages towards M2-type in colitis-associated colorectal cancer (CAC) that in response to commensal factors express the chemoattractant CCL-20. 3. CCL-20 recruits CCR-6-expressing lymphocytes comprising $B$ cells, regulatory T cells (Treg) and T cells expressing $\alpha \beta$ and $\gamma \delta$ T-cell receptors that impact on CAC progression. $B$ cells impact on macrophage polarisation, whereas CCR- $\sigma^{+} \gamma \delta$ T cells provide pathogenic IL-17. 4. IL-6 signalling in $\alpha \beta$ T cells releases them from Treg-mediated suppression to exert their effector functions such as T helper (Th)1 and Th17 responses. TAM, tumour-associated macrophage 
experiments assign obesity-induced IL-6 an unappreciated role in the CAC TME by regulating macrophage polarisation and lymphocyte recruitment.

\section{Discussion}

The steadily increasing obesity epidemic and the simultaneous incidence of obesity-associated comorbidities such as colorectal cancer requires a detailed understanding of molecular and cellular mechanisms that are affected to develop potential novel therapies. Here, we show that obesity exacerbates AOM/DSS-induced CAC via increased expression of inflammatory cytokines. However, our experimental set up was unable to address whether in obesity preneoplastic lesions occur earlier than in lean mice. We demonstrate that obesity-induced impairment of gut barrier function recruits and activates immune cells that promote CAC. In the colitis phase of CAC, IL-6-polarised M2-like macrophages express the chemokine CCL-20 that recruits CCR-6-expressing lymphocytes, further promoting CAC progression. Our in vitro experiments suggest that intestinal M2 macrophages express CCL-20 in colitis via exposure to commensal antigens such as LPS and not as a consequence of direct IL-6-activated transcriptional traits. Though IL-6-type cytokine-activated STAT3 can directly activate CCL-20 expression ${ }^{54}$, CCL-20 expression is mainly regulated via inflammatory signalling and nuclear factor kappa-light-chain-enhancer of activated B cells (NF-kB)-controlled transcription ${ }^{55-58}$. Given that LPS-activated NF-кBcontrolled gene expression in M1 macrophages has only minor effects on CCL-20 expression suggests extra transcription factors active in M2 macrophages that act in concert with LPS-induced NF- $\mathrm{KB}$ to increase CCL-20 expression. Hence, the substantially reduced Ccl20 expression in colons of Il6ra ${ }^{K O}$ mice is a consequence of collapsed M2-type polarisation of IL-6Ra-deficient macrophages in CAC, thereby compromising CCR-6-expressing lymphocyte recruitment in colitis to promote tumourigenesis. CCR-6-expressing B cells, $\gamma \delta$ T cells and Treg cells ${ }^{40,43}$ have either low or undetectable IL-6Ra expression underlining our observation that the protection against CAC in IL-6Ra-deficient mice is not a direct consequence of IL-6 action in these cells but is rather an indirect effect through compromised chemoattraction. Nevertheless, these cells might still be able to receive IL-6 signalling in control mice via IL-6-transsignalling mechanisms that we have avoided by using IL-6R $\alpha$-deficient mice devoid of both membrane and soluble signalling capacities. Despite the crucial role of direct IL-6 function on the approval of Treg-mediated suppression of $\alpha \beta \mathrm{T}$ cells, we estimate that Tregs also inhibit other immune cells by a similar mechanism as T-cell-specific inactivation of IL-6 signalling using CD4-Cre is unable to prevent CAC development. In line with this assumption, $\gamma \delta$ T cells in the colon were known to be suppressed via Tregs and $\gamma \delta \mathrm{T}$ cells are not affected by CD4-Cre-mediated recombination ${ }^{59}$. Although $\gamma \delta$ $\mathrm{T}$ cells can promote CRC via allocation of IL-17 to transformed tumour cells ${ }^{60,61}$, they are also able to restrain $\alpha \beta$ T-cell responses in cancer by providing inhibitory programmed cell death 1 ligand to $\alpha \beta \mathrm{T}$ cells ${ }^{62}$.

Collectively, our study assigns obesity-induced IL-6 as a modulator of the TME in CAC via macrophage polarisation and successive lymphocyte recruitment via the CCL-20/CCR-6 axis. In light of the steadily increasing obesity epidemic, novel treatment options to combat obesity-associated disorders are desperately required highlighting our study to interfere with inflammatory signalling and to inhibit corrupt cell types as important contribution to global health issues.

\footnotetext{
Methods

Animals. All animal procedures were in compliance with protocols approved by
} local government authorities (Bezirksregierung, Cologne, Germany Az: 8.87-
50.10.31.08.279, 84-02.04.2014.A211 and Mainz, Germany G16-1-072) and were in accordance with NIH guidelines. The experimental cohorts of mice (mus musculus) were females (if not stated differently) that were housed in four different specific pathogen free animal facilities under similar conditions. Mice were housed in single ventilated cages (IVCs, TypII long) in groups of three to five at $22-24^{\circ} \mathrm{C}$ in a 12 -hour-12-hour light-dark cycle (with lights on at 7:00 AM). The cages were implemented with bedding material (Lignocel FS14) and cotton nestlet material (Plexx, 14010). Mice were fed a NCD (Teklad Global Rodent T.2018.R12 Harlan) containing $53.5 \%$ carbohydrates, $18.5 \%$ protein and $5.5 \%$ fat $(12 \%$ of calories from fat) or, from 3 weeks of age, were fed a HFD (C1057; Altromin) containing $32.7 \%$ carbohydrates, $20 \%$ protein and $35.5 \%$ fat ( $55.2 \%$ of calories from fat). Food and water was available ad. libitum. The conditional II $6 r \alpha$ mouse strain served as control mice $\left(I I 6 r \alpha^{F l}\right)^{20}$. Mice bearing a complete $\left(I I 6 r \alpha^{K O}\right)^{22}$, myloidspecific $\left(I l 6 r \alpha^{m y l-K O}\right)^{19}$, intestinal epithelial-specific $\left(I l 6 r \alpha^{I E C-K O}\right)^{27}$ and T cellspecific $\left(I l 6 r \alpha^{T-K O}\right)^{34}$ knockout were generated and genotyped. For identifying the $I I 6 r \alpha^{F l}$ and the II $6 r \alpha^{K O}$ alleles PCR was performed with the following primers: 5GK12 (5'-CCGCGGGCGATCGCCTAGG-3'), 5IL6Rex3 (5'-CCAGAGGAGCCC AAGCTCTC-3') and 3IL6RA (5'-TAGGGCCCAGTTCCTTTAT-3'). The following primers were used to genotype the transgenes LysM-Cre LysMCre1 (5'-CTCTAGTCAGCCAGCAGCTG- $\left.3^{\prime}\right)$ and LysMCre2 (5'-ATGTTTAGCTGGCCCAAATGT-3'), Villin-Cre VilCre1 (5'-ACAGGCACTAAGGGAGCCAATG-3'), VilCre2 (5'-ATTGCAGGTCAGAAAGAGGTCACAG-3') and VilCre3 (5'-GTTCTTGCGAACCTCATCACTC- $\left.{ }^{\prime}\right)$ and CD4-Cre CD4Cre1 (5' CCCAACCAACAAGAGCTC-3') and CD4Cre2 (5'-CCCAGAAATGCCAGAT TACG-3'). C57BL/6 N (\#027) and C57BL/6 J (\#N/A) mice were obtained from Charles River, JHT ${ }^{51}$ and $C \mathrm{cr} 6^{\mathrm{KO}}(\# 013061)^{44}$ mice from Jackson laboratories. All animals were on a C57BL/6 N genetic background, except $C c r 6{ }^{\mathrm{KO}}$ and their control $C c r 6^{W T}$, they were on C57BL/6 J genetic background.

Induction of CAC in AOM/DSS model and cell depletion. 2.5\% AOM/DSS protocol: 8-week-old mice fed a NCD or a HFD were i.p. injected with $10 \mathrm{mg} / \mathrm{kg}$ body weight AOM (A5486, Sigma-Aldrich) at day 1 of the $2.5 \%$ AOM/DSS protocol (Supplementary Fig. 1f). The animals were exposed to three repetitive cycles of $2.5 \%$ DSS (MW $=36,000-50,000,0216011080$, MP Biomedicals) in the drinking water for 7 days from day 1-7, day 22-28 and day 43-49 (DSS water was refreshed every second day) to induce colitis. Animals were killed by $\mathrm{CO}_{2}$ with 17 weeks of age at day 62 for tumour analysis.

Mice that lost more than $20 \%$ of their body weight during the colitic phases were killed.

1.5\% AOM/DSS protocol: 8-week-old NCD or HFD-fed mice were i.p. injected with $10 \mathrm{mg} / \mathrm{kg}$ body weight AOM (A5486, Sigma-Aldrich) at day 1 of the $1.5 \%$ AOM/DSS protocol (Fig. 1c). At day 5 mice were exposed to $1.5 \%$ DSS (MW $=36,000-50,000,0216011080$, MP Biomedicals) in the drinking water for 5 days (DSS water was refreshed at day 7) and provided with normal drinking water from day 10 on until the end of the experiment at day 62. Animals were killed with 8 weeks at day 0 for non-colitic control tissue or at day 13 for colitic analysis or at day 62 for tumour analysis. The colon length was measured from caecum till the anus. The distal part of the colon was used for non-colitic and colitic analysis. At day 62 tumours were counted in a blinded fashion by number, size $(<2 \mathrm{~mm},>2 \mathrm{~mm})$ and percentage of the afflicted area of the distal colon. Tumour and non-tumour tissue from the distal colon was separated and used for tumour analysis.

To neutralise IL-6 and sIL-6R signalling, 8-week-old C57BL/6 animals were injected i.p. with $500 \mu \mathrm{g}$ anti-IL-6 antibody (BE0046. BioXCell) or $150 \mu \mathrm{g}$ sGP130Fc (provided by Christoph Garbers, Kiel University, Germany) at day 3 of the $1.5 \% \mathrm{AOM} / \mathrm{DSS}$ protocol, respectively.

To deplete $\gamma \delta$ TCR-expressing and Treg cells, $I I 6 r \alpha^{F l}$ and $I I 6 r \alpha^{K O}$ mice were injected with $200 \mu \mathrm{g}$ anti- $\gamma \delta$ antibody (UC7-13D5, eBioscience) or $1 \mathrm{mg}$ anti-CD25 antibody (BE0013, BioXCell) at day 3 of the AOM/DSS protocol, respectively. Control animals were injected with the same volume of PBS.

Endoscopy. Non-invasive endoscopy (TRICAM endoscope Karl Storz) was performed under either i.p. injection of ketamine/Rompun or inhalation narcosis using isoflurane with 13-week-old animals.

Analysis of body composition. Fat mass was determined via nuclear magnetic resonance (NMR Analyser minispeq mq7.5; Bruker Optik, Ettlingen, Germany) in 17-week-old mice.

Glucose-tolerance test. Glucose-tolerance tests were done with 7-8-week-old C57BL/6 mice. Mice were fasted overnight for $16 \mathrm{~h}$ and glucose concentrations in blood were measured after the fasting period. Then each animal received an i.p. injection of $20 \%$ glucose solution $(10 \mathrm{ml} / \mathrm{kg}$ body weight) and glucose concentrations in blood were measured after 15, 30, 60 and 120 min. Glucose concentrations in whole venous blood were measured with an automatic glucose monitor (Bayer Contour; Bayer). 
Analytical procedures. The concentrations of leptin and insulin in serum, CCL-20 in the supernatent of cultured BMDM and albumin in the faeces were measured by enzyme-linked immunosorbent assays, with mouse standards, according to manufacturer's guidelines.: Mouse Leptin ELISA (90030, Crystal Chem), Mouse Insulin ELISA (90080, Crystal Chem), Mouse CCL-20/MIP-3 alpha Quantikine ELISA Kit (MCC200, R\&D Systems) and Mouse Albumin ELISA Quantification set (E90-134, Bethyl).

\section{Generation of BMDMs. NCD-fed mice were killed by cervical} dislocation and bone marrow was isolated from femurs and tibias. Bone marrow cells were plated in RPMI-1640 medium (supplemented with $10 \%$ fecal calf serum (FCS), $1 \%$ glutamine, $1 \%$ penicillin-streptomycin and 10-50 ng/ml macrophage colony-stimulating factor (130-101-706, Miltenyi) and were allowed to differentiate for 7 days. At $24 \mathrm{~h}$ before all experiments, macrophage colony-stimulating factor was removed and cells were washed two times with sterile PBS. BMDMs were either stimulated $24 \mathrm{~h}$ with $20 \mathrm{ng} / \mathrm{ml}$ IFN $\gamma$ (130-094-048, Miltenyi) and $100 \mathrm{ng} / \mathrm{ml}$ LPS (Escherichia coli strain O55:B5; Sigma) or $20 \mathrm{ng} / \mathrm{ml} \mathrm{IL-4} \mathrm{(130-097-757,} \mathrm{Miltenyi)} \mathrm{and} 50 \mathrm{ng} / \mathrm{ml} \mathrm{IL-6}$ (130-096-685, Miltenyi) to obtain M1- and M2-polarised macrophages, respectively. Polarised M1 and M2 were washed with PBS and then stimulated for $8 \mathrm{~h}$ or $24 \mathrm{~h}$ with $50 \mathrm{ng} / \mathrm{ml} \mathrm{IL-6}$ and $10 \mathrm{ng} / \mathrm{ml} \mathrm{LPS}$.

MACS sorting. Immune cells and IECs from the colon were isolated according to recent protocol ${ }^{63}$. IECs were removed from the upper phase after the percoll gradient, washed twice with PBS containing $2 \%$ FCS. To purify macrophages, the percoll separated immune cells were used for magnetic separation with the anti-F4/ 80 MicroBeats UltraPure (130-110-443, Miltenyi) following the instruction manual. The IEC and macrophages were shock frozen in liquid nitrogen and kept at -80 degrees until further use.

Flow cytometry. Immune cells from the colon were isolated according to recent protocol ${ }^{63}$. The isolated immune cells were resuspended in FACS buffer (2\% FCS in PBS) and were passed through a $70-\mu \mathrm{m}$ strainer (BD Biosciences). Samples were analysed with following antibodies: anti-CD11b-PE (1:100 dilution, 101208, Biolegend), anti-CD11c-PE-Cy7 (1:100 dilution, 117318 , Biolegend), anti- $\gamma \delta$ TCR-APC (1:100 dilution, 118116, Biolegend), anti$\gamma \delta$ TCR-PE-Cy7 (1:100 dilution, 118123, Biolegend), anti- $\beta$ TCR-FITC (1:100 dilution, 109205, Biolegend), anti- $\beta$ TCR-PE (1:100 dilution, 553172, BD Biosciences), anti-CD45-V510 (1:100 dilution, 103137, Biolegend), anti-CD45-PECF594 (1:200 dilution, 562420, BD Biosciences), anti-CD45-APC (1:200 dilution, 103112, Biolegend), anti-CD90.2-PE-Cy7 (1:200 dilution, 140309, Biolegend), anti-CD3-PE-CF594 (1:200 dilution, 562332, BD Biosciences), anti-F4/80-APCCy7 (1:50 dilution, 123119, Biolegend), anti-CD19-FITC (1:100 dilution, 115505, Biolegend), anti-CCR-6-V421 (1:50 dilution, 129817, Biolegend), anti-IL-6R-PE (1:50 dilution, 115805, Biolegend) and LIVE/DEAD Fixable Violet Dead Cell Stain Kit or LIVE/DEAD Fixable Aqua Dead Cell Stain Kit (1:1000 dilution, L34955 and L34957, ThermoFisherScientific). Data were acquired on MACSQuant Analyzer (Miltenyi) and MACSQuant VYB (Miltenyi) and data were analysed with FlowJo software (Treestar).

Immunoblot analysis. Tissues were homogenised in protein lyses buffer with FastPrep-24 (MP Biomedicals) and centrifuged at $13,000 \mathrm{~g}$ for $1 \mathrm{~h}$ at $4^{\circ} \mathrm{C}$. Proteins were separated by SDS-polyacrylamide gel electrophoresis (10\%) and transferred to PVDF membranes (Bio-Rad). Membranes were probed with the following antibodies: Calnexin (1:5000 dilution, 208880, Calbiochem), PCNA (1:1000 dilution, D3H8P, 13110, CellSignalling).

Analysis of gene expression. Total RNA isolated from colons, tumour tissue, $\mathrm{T}$ cells, BMDMs and primary macrophages was analysed by qPCR. RNA was isolated from tissue and cells with an RNeasy Kit (74106, Qiagen) or Arcturus PicoPure (KIT0214, Applied Biosystems) and treated with

DNase. The RNA was reverse-transcribed with a High Capacity cDNA RT Kit (4368813, Applied Biosystems) and was amplified with TaqMan Gene Expression Master Mix (4369542, Applied Biosystems). The following primers from ThermoFisherscientific were used: Arg1 (Mm00475988_m1), Ccl5 (Mm01302428_m1), Ccl20 (Mm01268754_m1), Ccr1 (Mm00438260_s1), Ccr3 (Mm00515543_s1), Ccr6 (Mm99999114_s1), Cd3d (Mm00442746_m1), Cd19 (Mm00515420_m1), Emr1 (Mm00802529_m1), Foxp3 (Mm00475165_m1), Il1b (Mm01336189_m1), Il6 (Mm00446190_m1), Il10 (Mm00439616_m1), Il17 (Mm00439619_m1), Ill4r (Mm01275139_m1), Il6ra (Mm00439649_m1), Ifng (Mm01168134_m1), Nos2 (Mm00440485_m1), Tnf (Mm00443260_g1),

Tata box binding protein (Tbp) (Mm00446971_m1). Tbp was used as reference gene. Self-designed exon spanning probes for the constant region of the aTCR chain and the highly homologous 1,2 and $3 \gamma$ TCR were obtained from Eurogentec including forward and reverse primer as well as 5'-FAM/3'-TAMRA labelled probes: aTCR-fwd 5'-AAAACTGTGCTGGACATGAA-3', aTCR-rev 3'-CATCACAGGGAACGTCTGAA- ${ }^{\prime}$, and aTCR-probe $5^{\prime}$-FAMAGATATCTTGGCAGGT GAAGCTT-3' as well as $\gamma$ TCR-fwd $5^{\prime}$-CTGGCAAGATAAAAATGATGTG- ${ }^{\prime}$, $\gamma$ TCR-rev 3'-ACAGATGTTCTTCTAAGCAGA-5', and $\gamma$ TCR-probe $5^{\prime}$ ACCTCTGCCTACTACACCTACCT-3'. The expression of each specific mRNA analysed was adjusted for total RNA content by comparison with qPCR analysis of mRNA encoding Tbp. Results were calculated by the 'change-in-cycling-threshold' $(\Delta \mathrm{Ct})$ comparative method (as $2^{-\Delta \Delta \mathrm{Ct}}$ ). An ABI-PRISM 7900 HT Sequence Detection system (Applied Biosystems) was used for qPCR.

Microarray. For microarray expression data, RNA was isolated from tumour tissue of colons of $I l 6 r \alpha^{F l}$ and $I l 6 r \alpha^{K O}$ HFD-fed mice and further processed to be hybridised to GeneChip Mouse Gene 1.0 ST Arrays (Affymetrix) according to manufacturer's instructions ${ }^{19}$. Affymetrix Powertools and the robust multiarray average method were used for background correction, quantile normalisation and summarisation of the raw intensity values. $R$ software (version 2.13.1) along with the Bioconductor software package were used for the robust multiarray average method, statistical analysis (Student's $t$-test), calculation of the change in intensity and illustrations. Genes were considered significantly deregulated when they had a difference in expression with a $P$ value of $\leq 0.025$. Genes with a fold change of $\leq 1.5$ were removed from the data.

Immunohistochemistry. Colons were fixed in $4 \%$ formalin and embedded in paraffin. Paraffin sections of the colon were deparaffinised. Macrophages and $\mathrm{T}$ cells in the colon were stained with primary antibodies anti-F4/80 (1:500 dilution, MCA 4976, Serotec) or anti-CD3 (1:100 dilution, 5690, Abcam) and with secondary antibodies anti-rat-Biotin-SP (1:500 dilution, 112-065-003, Jackson) or AP-conjugated goat anti-rabbit (1:500 dilution, A3687, Sigma), respectively. Stainings were visualised with either ABC Kit Vectastain Elite (Vector Laboratories) and DAB substrate (Vector Laboratories) or Vector blue (Vector Laboratories). Colons were counterstained with haematoxylin or eosin according to standard protocol and imaged with AxioVision 4.2 (Carl Zeiss MicroImaging). Proliferating IEC were stained with anti-Ki67 (1:200 dilution, ab15580, Abcam), counterstained with 4',6-diamidino-2-phenylindole (DAPI) in the mounting medium (Vectashield) and imaged with Axio Imager 2 (Carl Zeiss MicroImaging). Immunoreactive density of Ki67 and DAPI-positive cells was set in relation to immunoreactive density of all DAPI-positive cells using Fuji.

Fluorescent immunohistochemistry of $4 \%$ paraformaldehyde-fixed cryosections was performed using the tyramide signal amplification (TSA) Fluorescence/ Cyanine 3 system (Fluorescein NEL701 A, Cyanine NEL 704 A, PerkinElmer) and a fluorescence microscope (IX70; Olympus or Leica SP8, Leica) using primary antibodies against F4/80 (1:100 dilution, AP10243P4-5, Acris), IgM biotin (1:100 dilution, 98673, Abcam), FoxP3 (1:100 dilution, 14-5773-82, eBioscience), CD3 (1:100 dilution, 16-0031-82, eBioscience), B220 (1:100 dilution, 553092, BD Bioscience), CCL-20-Allophycocyanin conjugated (1:50 dilution, IC760A, R + D Systems) and $\gamma \delta$ TCR 1:100 dilution, 13-58811-81, eBioscience) respectively. Nuclei were counterstained with Hoechst 3342 (Invitrogen) or mounting medium for fluorescence with 4,6-diamidino-2phenylindole (H-1200, Vector). In brief, cryosections were fixed in $4 \%$ PFA for 20 min followed by sequential incubation with methanol, avidin/biotin (Vector Laboratories) and protein blocking reagent (T144.1 Roti-ImmunoBlock, Roth) to eliminate unspecific background staining. Slides were then incubated overnight with primary antibody specific for the respective antigen. Subsequently, the slides were incubated for $30 \mathrm{~min}$ at room temperature with biotinylated secondary antibodies (1:300 dilution, 127-065-160 Jackson Immunoresearch and 554014, BD Pharmingen). All samples were finally treated with streptavidin-horseradish peroxidase and stained with TSA Fluorescence/Cyanine 3 systems according to the manufacturer's instructions (Fluorescein NEL701 A, Cyanine NEL 704 A, PerkinElmer).

Statistics. For the animal experiments in order to determine group size necessary for adequate statistical power, power analysis employing the programme $G^{\star}$ power was performed using preliminary data sets. Mice of the indicated genotype were assigned at random to groups. Mouse studies were performed in a blinded fashion. $P$ values were calculated with a two-tailed unpaired Student's $t$-test or for the comparison of more than two conditions with a one- or two-way ANOVA followed by Fisher LSD. $P$ values of 0.05 or less were considered significant, ${ }^{\star} p \leq 0.05,{ }^{* *} p \leq$ 0.01 and ${ }^{* * *} p \leq 0.001$.

Data availability. The authors declare that the data supporting the findings of this study are available within the article and its supplementary information files, or are available upon reasonable requests to the authors. Expression data are available at the Gene Express Omnibus database (http://www.ncbi.nlm.nih.gov/geo/) with accession number GSE111450.

Received: 3 February 2017 Accepted: 8 March 2018

Published online: 25 April 2018 


\section{References}

1. Bhaskaran, K. et al. Body-mass index and risk of 22 specific cancers: a population-based cohort study of 5.24 million UK adults. Lancet 384, 755-765 (2014).

2. Aleksandrova, $\mathrm{K}$. et al. Metabolic syndrome and risks of colon and rectal cancer: the European prospective investigation into cancer and nutrition study. Cancer Prev. Res. (Phila.) 4, 1873-1883 (2011).

3. Pischon, T. et al. Body size and risk of renal cell carcinoma in the European Prospective Investigation into Cancer and Nutrition (EPIC). Int. J. Cancer 118, 728-738 (2006)

4. Calle, E. E. \& Kaaks, R. Overweight, obesity and cancer: epidemiological evidence and proposed mechanisms. Nat. Rev. Cancer 4, 579-591 (2004).

5. Torre, L. A. et al. Global cancer statistics, 2012. CA Cancer J. Clin. 65, 87-108 (2015).

6. Schulz, M. D. et al. High-fat-diet-mediated dysbiosis promotes intestinal carcinogenesis independently of obesity. Nature 514, 508-512 (2014).

7. Beyaz, S. et al. High-fat diet enhances stemness and tumorigenicity of intestinal progenitors. Nature 531, 53-58 (2016).

8. Gregor, M. F. \& Hotamisligil, G. S. Inflammatory mechanisms in obesity Annu. Rev. Immunol. 29, 415-445 (2011).

9. Minehira, K. \& Tappy, L. Dietary and lifestyle interventions in the management of the metabolic syndrome: present status and future perspective. Eur. J. Clin. Nutr. 56, 1262 (2002).

10. $\mathrm{Xu}, \mathrm{H}$. et al. Chronic inflammation in fat plays a crucial role in the development of obesity-related insulin resistance. J. Clin. Invest. 112, 1821-1830 (2003).

11. Winer, D. A. et al. B cells promote insulin resistance through modulation of T cells and production of pathogenic IgG antibodies. Nat. Med. 17, 610-617 (2011).

12. Winer, S. et al. Normalization of obesity-associated insulin resistance through immunotherapy. Nat. Med. 15, 921-929 (2009).

13. Feuerer, M. et al. Lean, but not obese, fat is enriched for a unique population of regulatory $\mathrm{T}$ cells that affect metabolic parameters. Nat. Med. 15, 930-939 (2009).

14. Heinrich, P. C. et al. Principles of interleukin (IL)-6-type cytokine signalling and its regulation. Biochem. J. 374, 1-20 (2003).

15. Schuster, B. et al. Signaling of human ciliary neurotrophic factor (CNTF) revisited. The interleukin-6 receptor can serve as an alpha-receptor for CTNF. J. Biol. Chem. 278, 9528-9535 (2003).

16. Wolf, J., Rose-John, S. \& Garbers, C. Interleukin-6 and its receptors: a highly regulated and dynamic system. Cytokine 70, 11-20 (2014).

17. Kraakman, M. J. et al. Blocking IL-6 trans-signaling prevents high-fat dietinduced adipose tissue macrophage recruitment but does not improve insulin resistance. Cell Metab. 21, 403-416 (2015).

18. Timper, K. et al. IL-6 improves energy and glucose homeostasis in obesity via enhanced central IL-6 trans-signaling. Cell Rep. 19, 267-280 (2017)

19. Mauer, J. et al. Signaling by IL-6 promotes alternative activation of macrophages to limit endotoxemia and obesity-associated resistance to insulin. Nat. Immunol. 15, 423-430 (2014).

20. Wunderlich, F. T. et al. Interleukin-6 signaling in liver-parenchymal cells suppresses hepatic inflammation and improves systemic insulin action. Cell Metab. 12, 237-249 (2010).

21. Wunderlich, C. M., Hovelmeyer, N. \& Wunderlich, F. T. Mechanisms of chronic JAK-STAT3-SOCS3 signaling in obesity. JAKSTAT 2, e23878 (2013).

22. Gruber, S. et al. Obesity promotes liver carcinogenesis via Mcl-1 stabilization independent of IL-6Ralpha signaling. Cell Rep. 4, 669-680 (2013).

23. Murray, P. J. et al. Macrophage activation and polarization: nomenclature and experimental guidelines. Immunity 41, 14-20 (2014).

24. Mantovani, A. \& Locati, M. Tumor-associated macrophages as a paradigm of macrophage plasticity, diversity, and polarization: lessons and open questions. Arterioscler. Thromb. Vasc. Biol. 33, 1478-1483 (2013).

25. Grivennikov, S. et al. IL-6 and Stat 3 are required for survival of intestinal epithelial cells and development of colitis-associated cancer. Cancer Cell 15, 103-113 (2009).

26. Bollrath, J. et al. gp130-mediated Stat3 activation in enterocytes regulates cell survival and cell-cycle progression during colitis-associated tumorigenesis. Cancer Cell 15, 91-102 (2009).

27. $\mathrm{Hu}, \mathrm{B}$. et al. Microbiota-induced activation of epithelial IL-6 signaling links inflammasome-driven inflammation with transmissible cancer. Proc. Natl. Acad. Sci. USA 110, 9862-9867 (2013).

28. Becker, C. et al. IL-6 signaling promotes tumor growth in colorectal cancer. Cell Cycle 4, 217-220 (2005).

29. Reinecker, H. C. et al. Enhanced secretion of tumour necrosis factor-alpha, IL-6, and IL-1 beta by isolated lamina propria mononuclear cells from patients with ulcerative colitis and Crohn's disease. Clin. Exp. Immunol. 94, 174-181 (1993).

30. Neufert, C., Becker, C. \& Neurath, M. F. An inducible mouse model of colon carcinogenesis for the analysis of sporadic and inflammation-driven tumor progression. Nat. Protoc. 2, 1998-2004 (2007).
31. Morrow, R. J., Lawson, N., Hussaini, S. H. \& Asquith, P. The usefulness of faecal haemoglobin, albumin and alpha-1-antitrypsin in the detection of gastrointestinal bleeding. Ann. Clin. Biochem. 27, 208-212 (1990).

32. Becker, C., Fantini, M. C. \& Neurath, M. F. High resolution colonoscopy in live mice. Nat. Protoc. 1, 2900-2904 (2006).

33. Wunderlich, C. M. et al. Cutting edge: Inhibition of IL-6 trans-signaling protects from malaria-induced lethality in mice. J. Immunol. 188, 4141-4144 (2012).

34. Nish, S. A. et al. T cell-intrinsic role of IL-6 signaling in primary and memory responses. eLife 3, e01949 (2014).

35. Sica, A., Erreni, M., Allavena, P. \& Porta, C. Macrophage polarization in pathology. Cell Mol. Life. Sci. 72, 4111-4126 (2015).

36. Murray, P. J. \& Wynn, T. A. Protective and pathogenic functions of macrophage subsets. Nat. Rev. Immunol. 11, 723-737 (2011).

37. Liu, J. et al. Tumor-associated macrophages recruit CCR-6+regulatory $\mathrm{T}$ cells and promote the development of colorectal cancer via enhancing CCL-20 production in mice. PLoS ONE 6, e19495 (2011).

38. Liu, D. et al. IL-15 protects NKT cells from inhibition by tumor-associated macrophages and enhances antimetastatic activity. J. Clin. Invest. 122, 2221-2233 (2012).

39. Liu, B. et al. Tumor-associated macrophage-derived CCL-20 enhances the growth and metastasis of pancreatic cancer. Acta Biochim Biophys Sin $\mathbf{4 8}$ 1067-1074 (2016)

40. Liao, F. et al. CC-chemokine receptor- 6 is expressed on diverse memory subsets of T cells and determines responsiveness to macrophage inflammatory protein 3 alpha. J. Immunol. 162, 186-194 (1999).

41. Krzysiek, R. et al. Regulation of CCR- 6 chemokine receptor expression and responsiveness to macrophage inflammatory protein-3alpha/CCL-20 in human B cells. Blood 96, 2338-2345 (2000).

42. Kleinewietfeld, M. et al. CCR-6 expression defines regulatory effector/ memory-like cells within the CD25(+)CD4 + T-cell subset. Blood 105, 2877-2886 (2005).

43. Haas, J. D. et al. CCR-6 and NK1.1 distinguish between IL-17A and IFNgamma-producing gammadelta effector T cells. Eur. J. Immunol. 39, 3488-3497 (2009).

44. Kucharzik, T., Hudson, J. T. 3rd, Waikel, R. L., Martin, W. D. \& Williams, I. R CCR-6 expression distinguishes mouse myeloid and lymphoid dendritic cell subsets: demonstration using a CCR-6 EGFP knock-in mouse. Eur. J. Immunol. 32, 104-112 (2002).

45. Bowman, E. P. et al. Developmental switches in chemokine response profiles during B cell differentiation and maturation. J. Exp. Med. 191, 1303-1318 (2000).

46. Shimomura, Y. et al. Regulatory role of B-1 B cells in chronic colitis. Int. Immunol. 20, 729-737 (2008).

47. Mizoguchi, A., Mizoguchi, E., Takedatsu, H., Blumberg, R. S. \& Bhan, A. K. Chronic intestinal inflammatory condition generates IL-10-producing regulatory B cell subset characterized by CD1d upregulation. Immunity 16, 219-230 (2002)

48. Rosser, E. C. et al. Regulatory B cells are induced by gut microbiota-driven interleukin-1beta and interleukin-6 production. Nat. Med. 20, 1334-1339 (2014).

49. Kirkland, D. et al. B cell-intrinsic MyD88 signaling prevents the lethal dissemination of commensal bacteria during colonic damage. Immunity 36, 228-238 (2012)

50. Barr, T. A. et al. B cell depletion therapy ameliorates autoimmune disease through ablation of IL-6-producing B cells. J. Exp. Med. 209, 1001-1010 (2012).

51. Gu, H., Zou, Y. R. \& Rajewsky, K. Independent control of immunoglobulin switch recombination at individual switch regions evidenced through CreloxP-mediated gene targeting. Cell 73, 1155-1164 (1993).

52. Koenecke, $\mathrm{C}$. et al. In vivo application of $\mathrm{mAb}$ directed against the gammadelta TCR does not deplete but generates "invisible" gammadelta T cells. Eur. J. Immunol. 39, 372-379 (2009).

53. Nanno, M. et al. Exacerbating role of gammadelta $\mathrm{T}$ cells in chronic colitis of T-cell receptor alpha mutant mice. Gastroenterology 134, 481-490 (2008).

54. Arima, Y. et al. Regional neural activation defines a gateway for autoreactive T cells to cross the blood-brain barrier. Cell 148, 447-457 (2012).

55. Harant, H., Eldershaw, S. A. \& Lindley, I. J. Human macrophage inflammatory protein-3alpha/CCL-20/LARC/Exodus/SCYA20 is transcriptionally upregulated by tumor necrosis factor-alpha via a non-standard NF-kappaB site. FEBS Lett. 509, 439-445 (2001).

56. Chen, H. et al. Activation of STAT6 by STING is critical for antiviral innate immunity. Cell 147, 436-446 (2011)

57. Sugita, S. et al. Induction of macrophage-inflammatory protein-3alpha gene expression by TNF-dependent NF-kappaB activation. J. Immunol. 168, 5621-5628 (2002) 
58. Imaizumi, Y. et al. Human T cell leukemia virus type-I Tax activates human macrophage inflammatory protein-3 alpha/CCL-20 gene transcription via the NF-kappa B pathway. Int. Immunol. 14, 147-155 (2002).

59. Park, S. G. et al. T regulatory cells maintain intestinal homeostasis by suppressing gammadelta T cells. Immunity 33, 791-803 (2010).

60. $\mathrm{Wu}, \mathrm{P}$. et al. gammadeltaT17 cells promote the accumulation and expansion of myeloid-derived suppressor cells in human colorectal cancer. Immunity 40, 785-800 (2014).

61. Ramutton, T. et al. gammadelta $\mathrm{T}$ cells as a potential tool in colon cancer immunotherapy. Immunotherapy 6, 989-999 (2014).

62. Daley, D. et al. $\gamma \delta \mathrm{T}$ cells support pancreatic oncogenesis by restraining $\alpha \beta \mathrm{T}$ cell activation. Cell 166, 1485-1499 (2016). e1415.

63. Reissig, S., Hackenbruch, C. \& Hovelmeyer, N. Isolation of T cells from the gut. Methods Mol. Biol. 1193, 21-25 (2014).

\section{Acknowledgements}

The project was funded by CECAD, CMMC and DFG (SFB 670) funds. We are grateful to Jens Brüning, Sophie Steculorum and the MPI for Metabolism Research for discussions and resources. We thank Sarah Turpin and Linda Koch for proofreading the manuscript. This work would not have been possible without the technical support from Cathy Baitzel, Anke Lietzau, Heike Krämer, Pia Scholl and Nadine Spenrath.

\section{Author contributions}

C.M.W., P.J.A., A.L.O., P.A.-Q., M.C.V., M.-L.T., A.N. performed experiments and analysed data. A.W., C.G., S.T., J.M. and N.H. provided expertise and essential materials. C.M.W. and F.T.W. designed experiments and wrote the paper.

\section{Additional information}

Supplementary Information accompanies this paper at https://doi.org/10.1038/s41467018-03773-0.

Competing interests: The authors declare no competing interests.

Reprints and permission information is available online at http://npg.nature.com/ reprintsandpermissions/

Publisher's note: Springer Nature remains neutral with regard to jurisdictional claims in published maps and institutional affiliations.

(c) (i) Open Access This article is licensed under a Creative Commons Attribution 4.0 International License, which permits use, sharing, adaptation, distribution and reproduction in any medium or format, as long as you give appropriate credit to the original author(s) and the source, provide a link to the Creative Commons license, and indicate if changes were made. The images or other third party material in this article are included in the article's Creative Commons license, unless indicated otherwise in a credit line to the material. If material is not included in the article's Creative Commons license and your intended use is not permitted by statutory regulation or exceeds the permitted use, you will need to obtain permission directly from the copyright holder. To view a copy of this license, visit http://creativecommons.org/ licenses/by/4.0/.

(C) The Author(s) 2018 